\title{
EFFECT OF MINERAL AND BIOLOGICAL FERTILIZERS ON THE QUANTITATIVE AND THE QUALITATIVE PARAMETERS OF RICE CULTIVARS
}

\author{
ESFAHANI, A. A. ${ }^{1}-$ NiKNEJAD, Y. $.^{*}-$ FALLAH, H. ${ }^{1}-$ DASTAN, S. ${ }^{2}$ \\ ${ }^{1}$ Department of Agronomy, Ayatollah Amoli Branch, Islamic Azad University, Amol, Iran \\ ${ }^{2}$ Agricultural Biotechnology Research Institute of Iran (ABRII), Karaj, Iran \\ *Corresponding author \\ e-mail:yousofniknejad@gmail.com
}

(Received $25^{\text {th }}$ Nov 2017; accepted $30^{\text {th }}$ May 2018)

\begin{abstract}
The efficient use and management of organic matter are important aspects of sustainable agriculture. The quality of organic matter is relatively low in most of soils in Iran and the continuous use of mineral fertilizers may create environmental hazards. Therefore, the aim of this study was to evaluate the impact of combined use of mineral fertilizers along with organic manures on the rice production. The experiments were conducted in a randomized complete blocks design (RCBD) with factorial treatments, in three replicates, in two local rice cultivars in North Iran, in 2016. Two local rice cultivars, Tarom Hashemi and Tarom Mahalli, were chosen as the first factor; mineral fertilizers in four levels $100 \%$ recommended NPK, 75\% recommended nitrogen with $100 \%$ PK, 75\% recommended phosphorous with $100 \% \mathrm{NK}$, and $75 \%$ recommended potassium with $100 \% \mathrm{NP}$ were selected based on soil analysis and were arranged as the second factor; and three levels of bio-fertilizer 0 and 8 ton vermi-compost per hectare and 10 ton of rotted manure per hectare were the third factor. Results revealed that the highest paddy yield occurs with cultivar and bio-fertilizer interaction involving vermi-compost and manure usage for both cultivars. The highest paddy yield $\left(3962 \mathrm{~kg} \mathrm{ha}^{-1}\right)$ occurs with $100 \%$ recommended NPK and vermi-compost consumption. The highest amount of gel consistency for $c v$. Tarom Hashemi was observed with 100 of NPK and $75 \%$ of recommended nitrogen and manure consumption. The maximum amylose content for $c v$. Tarom Mahalli was produced with 100\% NPK recommended and manure consumption. Therefore, it can be concluded that by the integrated consumption of mineral and biological fertilizers, farmers can significantly reduce impacts of chemical fertilizers on the environment, and improve the qualitative and the quantitative parameters of rice cultivars with biological fertilizers.
\end{abstract}

Keywords: amylose content, grain length, paddy yield, randomized complete blocks design (RCBD), vermi-compost

\section{Introduction}

Rice (Oryza sativa L.) is one of the most important staple foods for nearly half of the world's population, most of them living in developing countries such as for example Iran. In Iran, rice is the second most widely consumed cereal next to wheat (PishgarKomleh et al., 2011). According to official statistics released by the food and agriculture organization (FAO), global area under rice cultivation has increased from 145 million hectares to over 160 million hectares during the past few years (FAO, 2016). Iran with 550 thousand hectares of paddy field and two million tons of white rice production has $0.4 \%$ of total rice production and cultivation area in the world. About $75 \%$ of the cultivation area lies to the northern strip, which includes the provinces of Guilan, Mazandaran and Golestan. The $25 \%$ of the cultivation area is situated in 13 additional provinces that possess different climates (Ministry of Jihad-e-Agriculture of Iran, 2016). 
Chemical fertilizers consumption is now widespread in Iran for supplementing plant nutrients. Manufacturers fabricate chemical fertilizers from inorganic materials, to increase the potential of natural and organic nutrients. Many heavy metals are present as impurities in varying amounts in such chemical fertilizers. The present planting system in Iran is totally dependent on the use of chemical fertilizers, pesticides, and growth regulators for enhancing crop productivity. However, the production gap in major food crops still remains even after intensive use of chemical fertilizers and pesticides for about 50-60 years in Iranian agriculture. In addition, agricultural lands have been reduced and there is a greater threat to soil resources due to changes in the global environment. Of late these problems have started attracting attention. Utilization of farmyard manure seems to be the most effective measure for this purpose (Ministry of Jihad-e-Agriculture of Iran, 2016).

Nitrogen, phosphorous, and potassium are the major elements essential for plant growth and development. To date use of chemical fertilizers has been confined mainly to the application of nitrogen and phosphorous and due attention has not been paid to potassium. Jogloy et al. (2006) reported that the application of animal manure plus chemical fertilizers gave the highest chlorophyll in Helianthus tuberosus, Xu et al. (2008) found that the application of organic manure with chemical fertilizers increases the yield more than using just chemical fertilizers because this combination increases nitrogen use efficiency of rice. Pazouki et al. (2017) demonstrate NPK have a high energy consumption and greenhouse gas emissions which caused the environmental pollution.

Deshpande and Devasenapathy (2010) observed that application of green manure along with farmyard manure (FYM) and poultry manure significantly increased protein and carbohydrate content in rice. Roy and Singh (2006) also reported similar results in barley with vermi-compost applications.

Metwally (2015) reported that the integrate use of organic materials and inorganic fertilizers significantly increase the yield and yield contributing characters of Egyptian rice cultivar Giza 179. The highest values of yield, yield parameters, and grain quality characters were observed when urea was used with compost as compared to mineral fertilizer alone. Mineral nitrogen combined with farmyard manure or compost could increase soil organic matter content. Moreover, integrated use of inorganic, organic, and bio-fertilizers positively affect rice yield and its attributes (Elekhtyar et al., 2016). Ghosh and Sharma (1999) reported that application of 10 tons manure per hectare combined with urea fertilizer has a beneficial effect on rice growth and yield in the farm conditions. In the second year, the yield obtained from the use of animal manure was similar to consumption of $40 \mathrm{~kg} \mathrm{~N} . \mathrm{ha}^{-1}$. Moreover, the highest grain yield was produced with application of manure and $40 \mathrm{~kg} \mathrm{~N} \cdot \mathrm{ha}^{-1}$. Regmi et al. (2002) found that rice yield with consumption of four ton farmyard manure per hectare and application of nitrogenphosphorous-potassium (NPK) was more than other treatments. Rekhi et al. (2004) reported that average rice grain yield with integrated application of farm yard manure and application of NPK fertilizer $\left(80,30\right.$, and $30 \mathrm{~kg} \mathrm{ha}^{-1}$ respectively) was higher than other treatments.

Hence, the aims of study were (1) to formulate organic fertilizers with different known organic sources like cow manure, poultry manure, and rice straw as well as husk, and (2) to study the effect of organic fertilizers alone and in combination with chemical fertilizers, and compare these with recommended doses of chemical fertilizers (i.e. NPK) on soil chemical properties under rice cultivation for two consecutive years. 


\section{Material and methods}

\section{Description of the experimental site}

Experiments were conducted in 2015 and 2016 in the paddy fields in the Iran Rice Research Institute between Alborz Mountains range and the Caspian Sea in Mazandaran and Guilan provinces in north of Iran. The experimental region is geographically situated at $36^{\circ} 31^{\prime} \mathrm{N}$ latitude and $52^{\circ} 27^{\prime}$ 'E longitude, west of the meridian, and is $78 \mathrm{~m}$ above sea level. The soil property of the experiment site is shown in Table 1. The most important climatic parameters of the three sites during the rice growth period are shown in Table 1. Solar radiation $\left(\mathrm{MJ} \mathrm{m}^{-2} \mathrm{~d}^{-1}\right)$ was estimated by the Srad_calc program and sunshine hours were estimated by the PP_calc program (https://sites.google.com/site/CropModeling).

Table 1. Description of climate parameters and soil properties in Amol region and description of name, origin and other characteristics of rice cultivars in the experiment

\begin{tabular}{|c|c|c|c|c|c|c|c|c|}
\hline Site & $\begin{array}{l}\text { Min. } \\
\text { temp. } \\
\left({ }^{\circ} \mathbf{C}\right)\end{array}$ & $\begin{array}{l}\text { Max. } \\
\text { temp. } \\
\left({ }^{\circ} \mathbf{C}\right)\end{array}$ & $\begin{array}{c}\text { Mean. } \\
\text { temp. } \\
\left({ }^{\circ} \mathbf{C}\right)\end{array}$ & $\begin{array}{c}\text { Evaporation } \\
(\mathbf{m m})\end{array}$ & $\begin{array}{l}\text { Rain } \\
(\mathbf{m m})\end{array}$ & $\begin{array}{c}\text { Mean } \\
\text { humidity } \\
(\%)\end{array}$ & $\begin{array}{c}\text { Mean } \\
\text { sunshine } \\
\text { hours }\end{array}$ & $\begin{array}{c}\text { Solar } \\
\text { radiation } \\
\left(\mathbf{M J ~ m}^{-2} \mathbf{d}^{-1}\right)\end{array}$ \\
\hline Apr. & 10.8 & 18.6 & 14.7 & 63.2 & 99.3 & 77 & 123.6 & 13.5 \\
\hline May & 16.4 & 24.8 & 20.6 & 85.9 & 41.4 & 78 & 140.9 & 15.9 \\
\hline Jun. & 19.9 & 27.8 & 23.8 & 121.8 & 24.6 & 80 & 232.8 & 21.1 \\
\hline Jul. & 22.3 & 30.7 & 26.5 & 130.2 & 39.6 & 79 & 203.0 & 19.7 \\
\hline Aug. & 22.5 & 33.1 & 27.8 & 142.3 & 11.4 & 76 & 232.5 & 20.2 \\
\hline Sep. & 21.6 & 31.0 & 26.3 & 113.9 & 88.5 & 65 & 193.0 & 16.5 \\
\hline $\begin{array}{c}\text { Mean } 15 \\
\text { years }\end{array}$ & 18.5 & 26.9 & 32.2 & 120.8 & 93.4 & 77.5 & 182.7 & 17.9 \\
\hline Cultivar & $\begin{array}{c}\text { Growth } \\
\text { condition }\end{array}$ & $\begin{array}{l}\text { Maturity } \\
\text { condition }\end{array}$ & \begin{tabular}{|c|} 
Growth \\
period
\end{tabular} & $\begin{array}{c}\text { Paddy yield } \\
\left(\mathrm{kg} \mathrm{ha}^{-1}\right)\end{array}$ & $\begin{array}{c}\text { Quality } \\
\text { condition }\end{array}$ & $\begin{array}{c}\text { Tolerance } \\
\text { to stress }\end{array}$ & Type & Origin \\
\hline $\begin{array}{c}c v . \text { Tarom } \\
\text { Hashemi }\end{array}$ & Tall plant & $\begin{array}{c}\text { Early } \\
\text { maturing }\end{array}$ & $\begin{array}{c}118 \\
\text { days }\end{array}$ & 4100 & $\begin{array}{l}\text { High } \\
\text { quality }\end{array}$ & $\begin{array}{c}\text { Little } \\
\text { sensitive }\end{array}$ & $\begin{array}{l}\text { Local } \\
\text { cultivar }\end{array}$ & Iran \\
\hline $\begin{array}{c}c v . \text { Tarom } \\
\text { Mahalli }\end{array}$ & Tall plant & $\begin{array}{c}\text { Early } \\
\text { maturing }\end{array}$ & $\begin{array}{c}123 \\
\text { days }\end{array}$ & 3600 & $\begin{array}{l}\text { High } \\
\text { quality }\end{array}$ & Sensitive & $\begin{array}{l}\text { Local } \\
\text { cultivar }\end{array}$ & Iran \\
\hline $\begin{array}{c}\text { Soil } \\
\text { properties }\end{array}$ & $\begin{array}{c}\text { Depth } \\
\text { (cm) }\end{array}$ & Texture & $\begin{array}{c}\mathbf{E C} \\
\left(\mathbf{d S m}^{-1}\right)\end{array}$ & pH & $\begin{array}{c}\text { Organic } \\
\text { matter } \\
(\%)\end{array}$ & $\begin{array}{c}\text { Phosphorus } \\
\left(\mathrm{mg} \mathrm{kg}^{-1}\right)\end{array}$ & $\mid \begin{array}{c}\text { Potassium } \\
\left(\mathrm{mg} \mathrm{kg}^{-1}\right)\end{array}$ & $\operatorname{CEC}(\%)$ \\
\hline $\begin{array}{l}\text { Amol } \\
\text { region }\end{array}$ & $0-30$ & $\begin{array}{l}\text { Clay } \\
\text { loam }\end{array}$ & 0.96 & 7.5 & 1.8 & 9.2 & 165 & 7.4 \\
\hline
\end{tabular}

\section{Description of the experiment}

The experiment was carried out based on a randomized complete blocks design (RCBD) with factorial treatment by three replications. Two local rice cultivars, Tarom Hashemi and Tarom Mahalli, were chosen as the first factor; mineral fertilizer in four levels $100 \%$ recommended NPK, 75\% recommended nitrogen with 100\% PK, 75\% recommended phosphorous with $100 \% \mathrm{NK}$, and $75 \%$ recommended potassium with $100 \%$ NP were selected based on soil analysis and arranged as the second factor; and three levels of bio-fertilizer 0 , and 8 ton vermi-compost per hectare and 10 ton rotted manure per hectare were the third factor. Nitrogen, phosphorous, and potassium 
fertilizers were applied according to the suggestions of the Iran Rice Research Institute and the results of soil analysis. The profile of the genotypes is shown in Table 1. Description of the site subsections (a map of the experimental region and a photo of the experimental blocks) are showed in Figures 1 and 2.

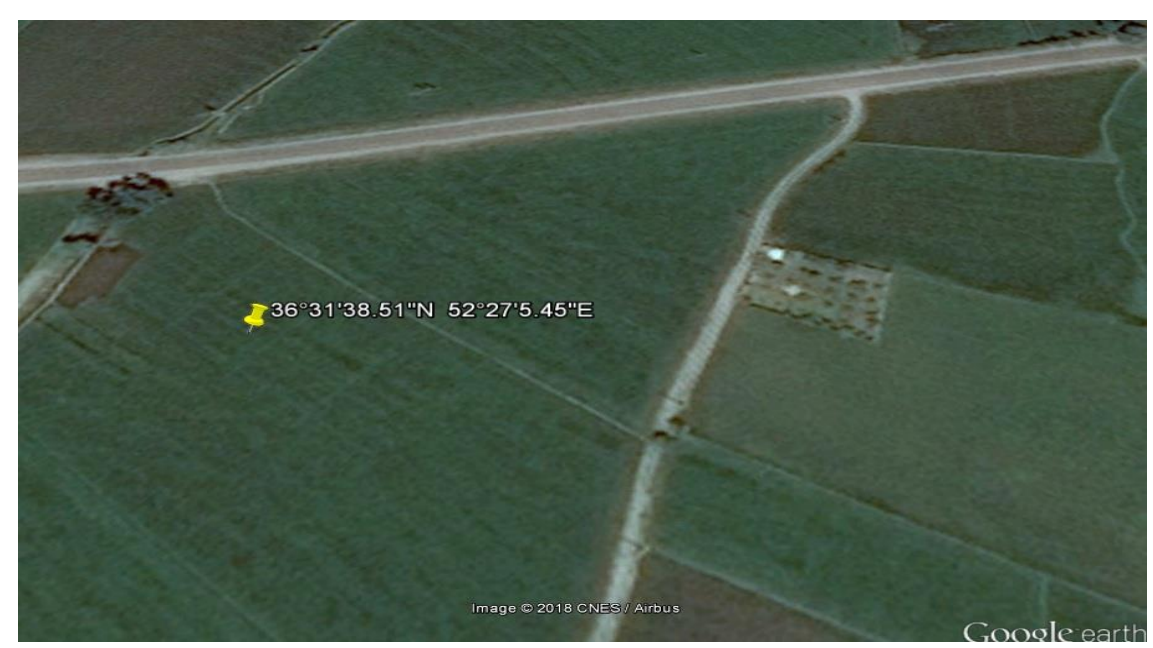

Figure 1. Description of the site subsections (a map of the experimental region)

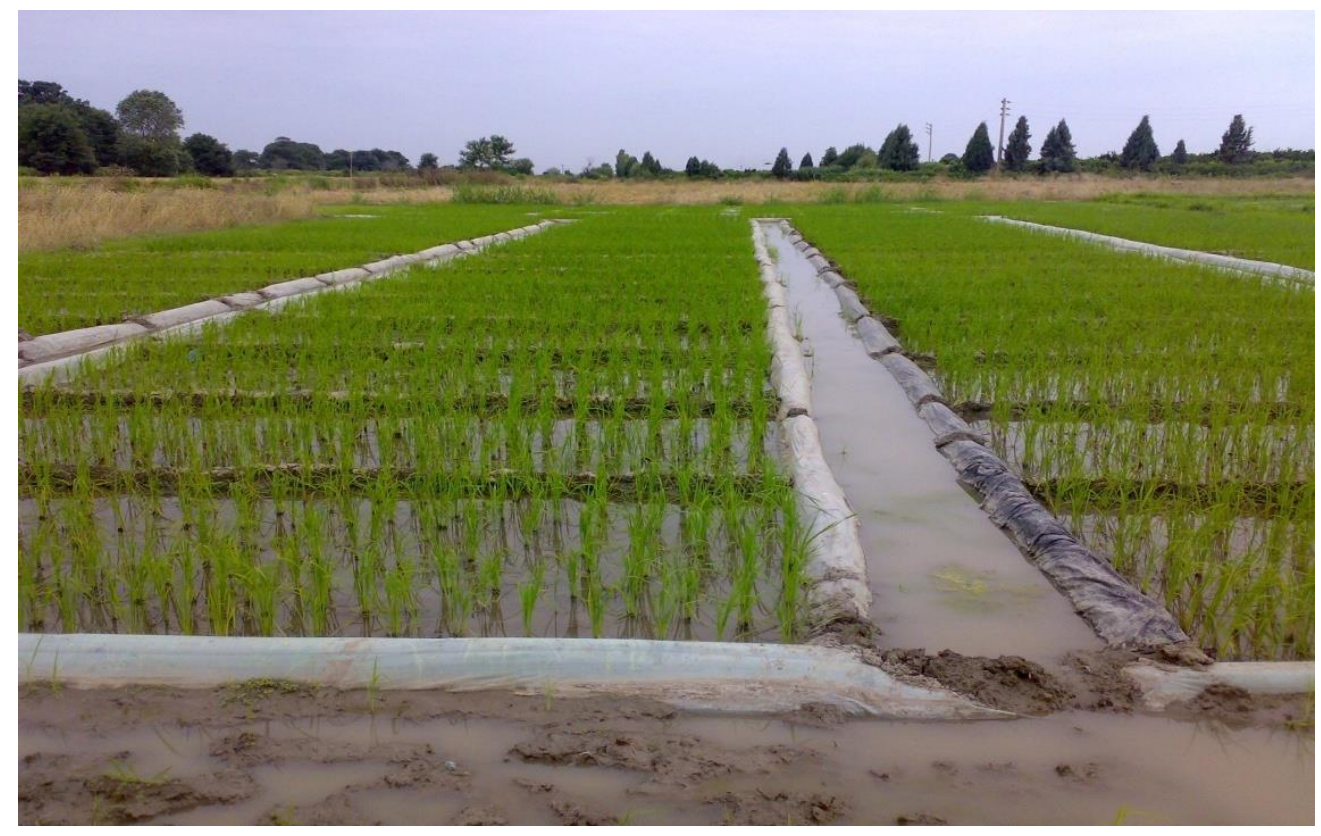

Figure 2. Description of the site subsection (a photo of the experimental blocks)

Considering the regional climates, seedlings were transplanted in 3-4 leaf stages. Considering the type of the cultivar, the transplanting operation was done in all the three regions with similar situations. The size of each plot was decided as $3 \times 4 \mathrm{~m}^{2}$, and planting density was 16 plants per square meter by $25 \times 25 \mathrm{~cm}^{2}$ arrangement. All of the phosphorus and one third of the nitrogen fertilizer were used as basal in paddy field preparation stage. Two thirds of the nitrogen fertilizer was used as top-dressing in 
panicle initiation and full heading stages. Sixty percent of potassium fertilizer was used as basal and the remaining amount was used as top-dressing in tillering and panicle initiation stages (splitting to $20 \%$ in each stage). Moreover, depth of irrigation water was set at five centimetres according to agricultural principles. In order to prevent the growth of weeds and the mixing of water and fertilizer in paddy fields, nylon plastic covers were put at the borders up to the depth of $30 \mathrm{~cm}$.

Crop protection practices, such as irrigation, weeding, pests and disease controls, and fertilization, were done in the experiment paddy field based on technical instruction of the Iran Rice Research Institute. Other agricultural operations and field management were done according to the Standard Evaluation System (SES) of the International Rice Research Institute (IRRI).

\section{Evaluation traits}

\section{Agronomical traits}

During the growth period, after the removal of marginal effect in each site, traits were randomly measured according to the Standard Evaluation System (SES) of the International Rice Research Institute (IRRI). Thus, 10 hills per plant were randomly selected from each experimental plot and their average was analyzed.

In order to quantitatively determine the morphological traits, sampling was done 30 days after full heading stage from 12 stems selected from four hills per plant in each experimental plot. Number of tillers per hill was counted by 12 hills per plant in each experimental plot. The number of spikelet per panicle and number of filled spikelet per panicle were measured by counting from 15 panicles in each experimental plot.

Paddy yield and biological yield were measured by harvesting hills from four square meters in the middle part of each plot based on $12 \%$ moisture. From the relationship between paddy yield and biological yield, the harvest index was calculated and expressed in percentage.

\section{Determination of grain quality traits}

The qualitative traits of rice grain such as grain amylose content, gelatinization temperature, gel consistency, grain length before and after baking, grain width after cooking, grain elongation, conversion efficiency, aroma, brown rice, and healthy and broken grain percentage were measured by the Standard Evaluation System (SES) of the International Rice Research Institute (IRRI).

\section{Statistical analysis}

Aftat first, normalization and Kaiser-Meyer-Olkin (KMO) were test. After that, all the investigated data (agronomical and grain quality traits) were statistically analyzed for analysis of variance (ANOVA) using SAS ver. 9.1 software program. The averages so obtained were compared by Least Significant Differences (LSD) test at 5\% probability level.

\section{Results and discussion}

In order to evaluate these traits, the obtained data was analyzed using variance homogeneity test by the Bartlett method. The results showed that the traits were not 
significant in Bartlett's test. Therefore, data analysis was performed based on combined analysis.

\section{Agronomical traits}

Data concerning quantitative parameters is presented in Table 2. Combined variance analysis of agronomical traits indicates that filling all of these traits demonstrates significant difference under the effect of year, mineral fertilizer, and bio-fertilizer at $1 \%$ and $5 \%$ probability level respectively. But, under the effect of cultivar, only number of tiller per hill, and number of filled spikelet per panicle revealed significant differences. Moreover, at double interaction of year and cultivar, number of tiller per hill was significant at $1 \%$ probability. But, number of spikelet per panicle and number of filled spikelet per panicle showed significant difference $(\mathrm{P} \leq 0.05)$ at double interaction of cultivar and mineral fertilizer. Only number of tiller per hill appeared significant under the effect of cultivar and bio-fertilizer interaction. A long with number of tiller per hill, number of spikelet per panicle and number of filled spikelet per panicle showed significant differences $(\mathrm{P} \leq 0.05 ; \mathrm{P} \leq 0.01)$ at double interaction of mineral fertilizer and bio-fertilizer (Table 2). At triple interaction of year with cultivar and mineral fertilizer, significant differences occurred at 5\% probability level for number of spikelet per panicle and number of filled spikelet per panicle. Moreover, triple interaction of cultivar with mineral fertilizer and bio-fertilizer had a significant effect $(\mathrm{P} \leq 0.05 ; \mathrm{P} \leq 0.01)$ on number of spikelet per panicle, number of filled spikelet per panicle, and number of blank spikelet per panicle (Table 2).

Table 2. Combined analysis of variance (ANOVA) of agronomical traits, quantities yield and harvest index of rice cultivar under chemical fertilizer and bio-fertilizer

\begin{tabular}{|c|c|c|c|c|c|c|c|}
\hline S.O.V. & DF & $\begin{array}{l}\text { Number } \\
\text { of tiller } \\
\text { per hill }\end{array}$ & $\begin{array}{c}\text { Number of } \\
\text { spikelet } \\
\text { per panicle }\end{array}$ & $\begin{array}{l}\text { Number of } \\
\text { filled spikelet } \\
\text { per panicle }\end{array}$ & $\begin{array}{c}\text { Number of } \\
\text { blank spikelet } \\
\text { per panicle }\end{array}$ & Paddy yield & $\begin{array}{c}\text { Harvest } \\
\text { index }\end{array}$ \\
\hline Year $(Y)$ & 1 & $9.51^{*}$ & $457.57^{* *}$ & $671.80^{* *}$ & $20.51^{* *}$ & $44519.59^{\text {ns }}$ & $0.07^{\mathrm{ns}}$ \\
\hline $\mathrm{Y}(\mathrm{R})$ & 4 & 11.19 & 24.12 & 31.44 & 1.75 & 37618.30 & 2.08 \\
\hline Variety (V) & 1 & $91.84^{* *}$ & $43.79^{\mathrm{ns}}$ & $72.92^{*}$ & $3.55^{\mathrm{ns}}$ & $1694166.90^{* *}$ & $2.14^{\mathrm{ns}}$ \\
\hline Chemical F. (C) & 3 & $22.90^{* *}$ & $435.32^{* *}$ & $617.54^{* *}$ & $17.62^{* *}$ & $932104.34^{* *}$ & $1.80^{\mathrm{ns}}$ \\
\hline Bio-fertilizer (B) & 2 & $124.53^{* *}$ & $941.60^{* *}$ & $1471.26^{* *}$ & $61.66^{* *}$ & $2761965.98^{* *}$ & $1.09^{\mathrm{ns}}$ \\
\hline $\mathrm{Y} \times \mathrm{V}$ & 1 & $9.51^{*}$ & $18.84^{\mathrm{ns}}$ & $34.45^{\mathrm{ns}}$ & $2.34^{\mathrm{ns}}$ & $4764.22^{\mathrm{ns}}$ & $0.54^{\mathrm{ns}}$ \\
\hline $\mathrm{Y} \times \mathrm{C}$ & 3 & $3.73^{\mathrm{ns}}$ & $15.42^{\mathrm{ns}}$ & $25.93^{\mathrm{ns}}$ & $1.78^{\mathrm{ns}}$ & $40921.87^{\mathrm{ns}}$ & $0.02^{\mathrm{ns}}$ \\
\hline $\mathrm{Y} \times \mathrm{B}$ & 2 & $2.19^{\mathrm{ns}}$ & $2.92^{\mathrm{ns}}$ & $9.96^{\mathrm{ns}}$ & $2.66^{\mathrm{ns}}$ & $22856.21^{\mathrm{ns}}$ & $3.18^{\mathrm{ns}}$ \\
\hline $\mathrm{V} \times \mathrm{C}$ & 3 & $0.99^{\text {ns }}$ & $70.97^{*}$ & $73.19^{*}$ & $1.43^{\mathrm{ns}}$ & $62077.76^{\mathrm{ns}}$ & $4.87^{\mathrm{ns}}$ \\
\hline $\mathrm{V} \times \mathrm{B}$ & 2 & $5.53^{*}$ & $5.62^{\mathrm{ns}}$ & $13.12^{\mathrm{ns}}$ & $1.65^{\mathrm{ns}}$ & $139634.07^{*}$ & $5.64^{\mathrm{ns}}$ \\
\hline $\mathrm{C} \times \mathrm{B}$ & 6 & $8.19^{* *}$ & $58.71^{*}$ & $65.93^{*}$ & $5.86^{*}$ & $80426.99^{*}$ & $19.92^{* *}$ \\
\hline $\mathrm{Y} \times \mathrm{V} \times \mathrm{C}$ & 3 & $3.73^{\mathrm{ns}}$ & $71.65^{*}$ & $74.01^{*}$ & $0.16^{\mathrm{ns}}$ & $25564.56^{\mathrm{ns}}$ & $0.90^{\mathrm{ns}}$ \\
\hline $\mathrm{Y} \times \mathrm{V} \times \mathrm{B}$ & 2 & $2.19^{\mathrm{ns}}$ & $11.48^{\mathrm{ns}}$ & $14.17^{\mathrm{ns}}$ & $0.21^{\mathrm{ns}}$ & $1597.59^{\mathrm{ns}}$ & $0.79^{\mathrm{ns}}$ \\
\hline $\mathrm{Y} \times \mathrm{C} \times \mathrm{B}$ & 6 & $2.19^{\mathrm{ns}}$ & $32.25^{\mathrm{ns}}$ & $31.48^{\mathrm{ns}}$ & $0.77^{\mathrm{ns}}$ & $8247.64^{\mathrm{ns}}$ & $0.67^{\mathrm{ns}}$ \\
\hline $\mathrm{V} \times \mathrm{C} \times \mathrm{B}$ & 6 & $3.79^{\mathrm{ns}}$ & $81.12^{* *}$ & $69.61^{*}$ & $5.39^{*}$ & $25175.47^{\mathrm{ns}}$ & $5.14^{\mathrm{ns}}$ \\
\hline $\mathrm{Y} \times \mathrm{V} \times \mathrm{C} \times \mathrm{B}$ & 6 & $2.19^{\mathrm{ns}}$ & $11.32^{\mathrm{ns}}$ & $11.76^{\mathrm{ns}}$ & $1.69^{\mathrm{ns}}$ & $6249.49^{\mathrm{ns}}$ & $0.84^{\mathrm{ns}}$ \\
\hline Error & 92 & 2.32 & 24.59 & 24.88 & 2.12 & 29014.78 & 3.43 \\
\hline C.V. (\%) & - & 7.81 & 4.86 & 5.40 & 15.10 & 4.90 & 3.90 \\
\hline
\end{tabular}

$\mathrm{ns}, *$ and $* *$ : non significant and significant in $5 \%$ and $1 \%$ probability level, respectively 
At double interaction of year and cultivar, most tillers per hill were measured in the first year for $c v$. Tarom Hashemi. The least number of tillers per hill were produced in both years for $c v$. Tarom Hashemi (Fig. 3). Furthermore, at double interaction of cultivar and bio-fertilizer the maximum number of tiller per hill was obtained for $c v$. Tarom Hashemi with 8 ton vermi-compost per hectare; the maximum tiller for $c v$. Tarom Mahalli was produced with 8 ton vermi-compost per hectare and 10 ton rotted manure per hectare. The lowest number of tiller per hill for both cultivars was obtained in control treatment (Fig. 4).

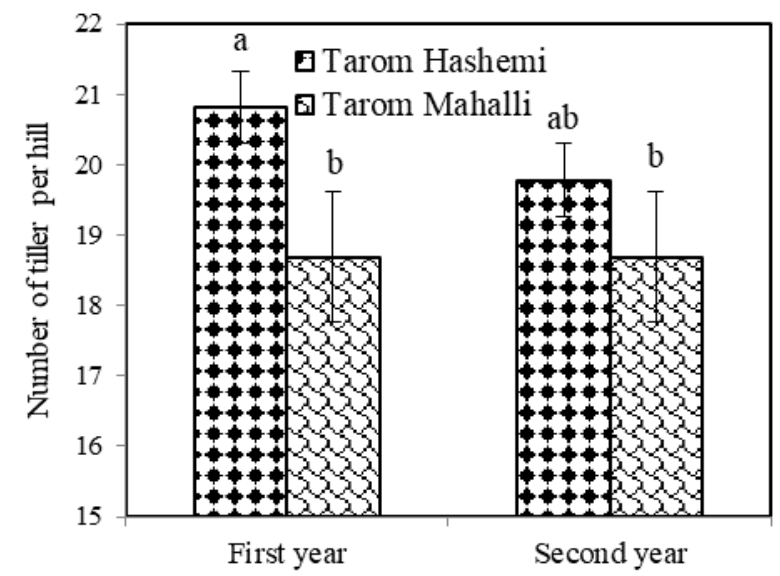

Figure 3. Double interaction of year and cultivar on the number of tiller per hill

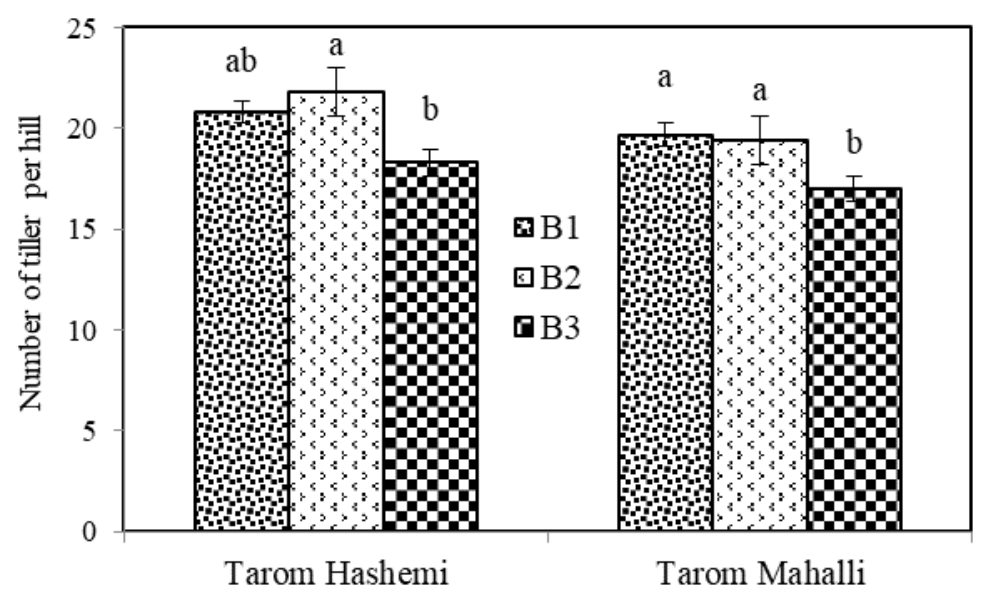

Figure 4. Mean comparison of cultivar and bio-fertilizer on the number of tiller per hill by slice interaction. $\left(B_{1}, B_{2}\right.$, and $B_{3}: 0$ and 8 ton vermi-compost per hectare and 10 ton of rotted manure per hectare, respectively)

Slice interaction of cultivar, mineral fertilizer, and bio-fertilizer demonstrated that the highest spikelets per panicle and number of filled spikelets per panicle were obtained for $c v$. Tarom Hashemi with application of $100 \%$ recommended NPK, and 8 ton vermi-compost, and 10 ton manure per hectare, and interaction of $75 \%$ recommended phosphorous with 10 ton vermi-compost consumption. The findings of $c v$. Tarom Mahalli cultivar were different from $c v$. Tarom Hashemi: the highest number 
of spikelets per panicle and number of filled spikelets per panicle were observed by application of $100 \%$ recommended NPK, and $75 \%$ recommended $\mathrm{N}$ and K. The least number of spikelet per panicle were obtained for both cultivars in the control level of bio-fertilizer with 75\% recommended N and K. Moreover, for $c v$. Tarom Hashemi $75 \%$ recommended phosphorous with control treatment of bio-fertilizer showed lowest spikelets per panicle. The lowest number of filled spikelets per panicle for $c v$. Tarom Hashemi was obtained at $75 \%$ recommended nitrogen and bio-fertilizer control treatment. For $c v$. Tarom Hashemi, the lowest filled spikelet per panicle was produced with application of $75 \%$ recommended $\mathrm{N}$ and $\mathrm{K}$ in bio-fertilizer control treatment (Table 6). The result was varied in terms of number of blank spikelets per panicle. The highest number of blank spikelets per panicle for $c v$. Tarom Hashemi were measured with application of $75 \%$ recommended $\mathrm{N}$ and $\mathrm{K}$ in bio-fertilizer control treatment. But the blankest spikelets for $c v$. Tarom Mahalli was obtained at all levels of mineral fertilizer in bio-fertilizer control treatment (Table 6)

\section{Quantitative yield and harvest index}

Combined statistical analysis of the data revealed that paddy yield was significant under the cultivar, mineral fertilizer, and bio-fertilizer treatment at $1 \%$ probability level. Also, paddy yield was statistically significant $(\mathrm{P} \leq 0.05)$ at interaction of cultivar and bio-fertilizer. Moreover, paddy yield, and harvest index showed significant differences at double interaction of mineral fertilizer and bio-fertilizer (Table 2). Mean comparison showed that the highest paddy yield for cultivar and bio-fertilizer interaction belonged to 8 ton vermi-compost and 10 ton manure per hectare for both cultivars. Moreover, the least paddy yield for both cultivars was produced at bio-fertilizer control level (Fig. 5). At double interaction of mineral fertilizer and bio-fertilizer, the highest paddy yield was produced for $100 \%$ recommended NPK with 8 ton vermi-compost per hectare. The least paddy yield was obtained upon application of $75 \%$ recommended $\mathrm{P}$ and $\mathrm{K}$ and at biofertilizer control level (Table 3). At double interaction of mineral fertilizer and biofertilizer, the highest harvest index was measured with $75 \%$ recommended phosphorous usage in bio-fertilizer control treatment. The least harvest index was obtained with $75 \%$ recommended phosphorous usage and 10 ton manure per hectare (Table 3).

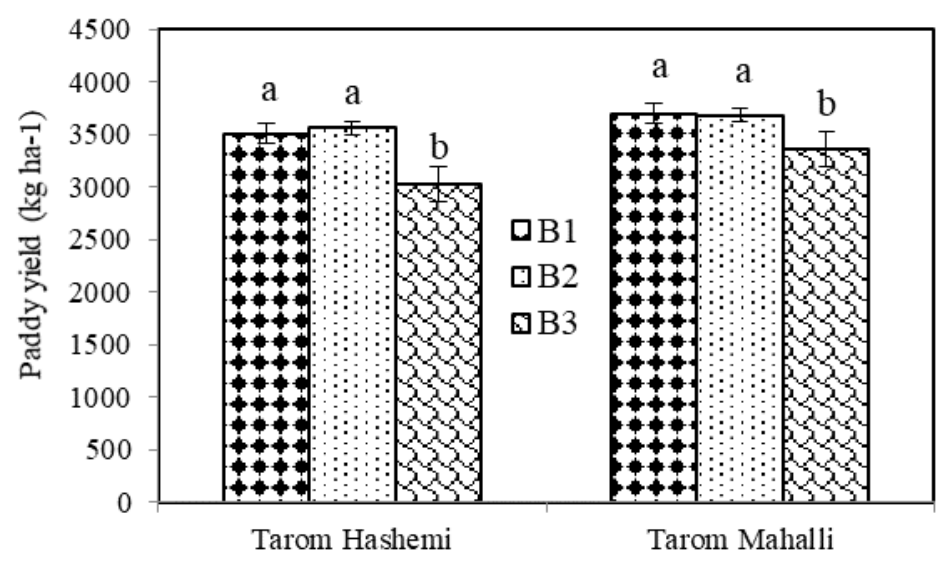

Figure 5. Mean comparison of cultivar and bio-fertilizer on paddy yield by slice interaction. $\left(B_{1}, B_{2}\right.$, and $B_{3}: 0$ and 8 ton vermi-compost per hectare and 10 ton of rotted manure per hectare, respectively) 
Table 3. Mean comparison of chemical and biological fertilizers on the number of tiller per hill, paddy yield and harvest index by slice interaction

\begin{tabular}{c|c|c|c}
\hline Interaction & Number of tiller per plant & Paddy yield & Harvest index \\
\hline $\mathrm{C}_{1} \mathrm{~B}_{1}$ & $21.50 \mathrm{a}$ & $3784 \mathrm{ab}$ & $47.27 \mathrm{~b}$ \\
$\mathrm{C}_{1} \mathrm{~B}_{2}$ & $22.67 \mathrm{a}$ & $3962 \mathrm{a}$ & $48.06 \mathrm{ab}$ \\
$\mathrm{C}_{1} \mathrm{~B}_{3}$ & $17.83 \mathrm{c}$ & $3303 \mathrm{bc}$ & $48.27 \mathrm{ab}$ \\
$\mathrm{C}_{2} \mathrm{~B}_{1}$ & $19.50 \mathrm{abc}$ & $3677 \mathrm{ab}$ & $48.43 \mathrm{ab}$ \\
$\mathrm{C}_{2} \mathrm{~B}_{2}$ & $19.75 \mathrm{abc}$ & $3631 \mathrm{ab}$ & $46.79 \mathrm{bc}$ \\
$\mathrm{C}_{2} \mathrm{~B}_{3}$ & $17.42 \mathrm{c}$ & $3264 \mathrm{bcd}$ & $46.83 \mathrm{bc}$ \\
$\mathrm{C}_{3} \mathrm{~B}_{1}$ & $19.25 \mathrm{abc}$ & $3452 \mathrm{bc}$ & $45.80 \mathrm{c}$ \\
$\mathrm{C}_{3} \mathrm{~B}_{2}$ & $20.33 \mathrm{ab}$ & $3451 \mathrm{bc}$ & $47.43 \mathrm{~b}$ \\
$\mathrm{C}_{3} \mathrm{~B}_{3}$ & $18.17 \mathrm{bc}$ & $3123 \mathrm{~d}$ & $49.17 \mathrm{a}$ \\
$\mathrm{C}_{4} \mathrm{~B}_{1}$ & $20.67 \mathrm{ab}$ & $3508 \mathrm{abc}$ & $48.61 \mathrm{ab}$ \\
$\mathrm{C}_{4} \mathrm{~B}_{2}$ & $19.67 \mathrm{abc}$ & $3452 \mathrm{bc}$ & $47.34 \mathrm{~b}$ \\
$\mathrm{C}_{4} \mathrm{~B}_{3}$ & $17.17 \mathrm{c}$ & $3107 \mathrm{~d}$ & $46.55 \mathrm{bc}$ \\
\hline
\end{tabular}

Values within a column followed by same letter are not significantly different at $\mathrm{LSD}(\mathrm{P} \leq 0.05)$

$\mathrm{C}_{1}, \mathrm{C}_{2}, \mathrm{C}_{3}$, and $\mathrm{C}_{4}$ : $100 \%$ recommended NPK, $75 \%$ recommended nitrogen with $100 \% \mathrm{PK}, 75 \%$ recommended phosphorous with $100 \% \mathrm{NK}$, and $75 \%$ recommended potassium with $100 \% \mathrm{NP}$, respectively

$\mathrm{B}_{1}, \mathrm{~B}_{2}$, and $\mathrm{B}_{3}: 0$ and 8 ton vermi-compost per hectare and 10 ton of rotted manure per hectare, respectively

\section{Qualitative parameters}

Combined variance analysis of qualitative parameters indicates that brown rice, hard husk, white rice, healthy grain, milling efficiency, grain width before cooking, gelatinization temperature, gel consistency, and amylose content showed significant difference under the effect of cultivar at $1 \%$ and 5\% probability level. Moreover, white rice, soft husk, healthy grain, milling efficiency, grain length before and after cooking, grain elongation, aroma, gelatinization temperature, gel consistency, and amylose content were statistically significant $(\mathrm{P} \leq 0.05 ; \mathrm{P} \leq 0.01)$ under the effect of mineral fertilizer (Table 4). Similarly, soft husk, healthy grain, grain length before cooking, gelatinization temperature, gel consistency, and amylose content were significant at 5\% and $1 \%$ probability level under the effect of bio-fertilizer. Moreover, all qualitative traits except grain width before cooking had significant difference statistically $(\mathrm{P} \leq$ $0.05 ; \mathrm{P} \leq 0.01)$ at triple interaction of cultivar, mineral fertilizer, and bio-fertilizer (Table 5).

Table 4. Combined analysis of variance (ANOVA) of chlorophyll content and nitrogen, phosphorous and potassium content of grain in rice cultivar under chemical fertilizer and bio-fertilizer

\begin{tabular}{c|c|c|c|c|c|c|c|c}
\hline S.o.v. & DF & $\begin{array}{c}\text { Brown } \\
\text { rice }\end{array}$ & $\begin{array}{c}\text { Hard } \\
\text { husk }\end{array}$ & $\begin{array}{c}\text { White } \\
\text { rice }\end{array}$ & $\begin{array}{c}\text { Soft } \\
\text { husk }\end{array}$ & $\begin{array}{c}\text { Health } \\
\text { grain }\end{array}$ & $\begin{array}{c}\text { Broken } \\
\text { grain }\end{array}$ & $\begin{array}{c}\text { Milling } \\
\text { efficiency }\end{array}$ \\
\hline Year (Y) & 1 & $17.49^{* *}$ & $17.49^{* *}$ & $6.73^{* *}$ & $45.89^{* *}$ & $0.71^{\text {ns }}$ & $17.49^{* *}$ & $6.73^{* *}$ \\
Y (R) & 4 & 0.07 & 0.07 & 1.30 & 1.66 & 1.10 & 0.07 & 1.30 \\
Variety (V) & 1 & $16.22^{* *}$ & $16.22^{* *}$ & $14.48^{* *}$ & $0.05^{\text {ns }}$ & $11.92^{* *}$ & $16.22^{* *}$ & $14.48^{* *}$ \\
Chemical F. (C) & 3 & $0.40^{\text {ns }}$ & $0.40^{\text {ns }}$ & $3.57^{* *}$ & $3.59^{* *}$ & $6.06^{* *}$ & $0.40^{\text {ns }}$ & $3.57^{* *}$ \\
Bio-fertilizer (B) & 2 & $0.24^{\text {ns }}$ & $0.24^{\text {ns }}$ & $0.50^{\text {ns }}$ & $1.42^{*}$ & $1.46^{*}$ & $0.24^{\text {ns }}$ & $0.50^{\text {ns }}$ \\
\hline
\end{tabular}




\begin{tabular}{c|c|c|c|c|c|c|c|c}
\hline $\mathrm{Y} \times \mathrm{V}$ & 1 & $3.56^{* *}$ & $3.56^{* *}$ & $2.87^{*}$ & $0.04^{\mathrm{ns}}$ & $2.52^{*}$ & $3.56^{* *}$ & $2.87^{*}$ \\
$\mathrm{Y} \times \mathrm{C}$ & 3 & $0.11^{\mathrm{ns}}$ & $0.11^{\mathrm{ns}}$ & $0.09^{\mathrm{ns}}$ & $0.33^{\mathrm{ns}}$ & $1.53^{*}$ & $0.11^{\mathrm{ns}}$ & $0.09^{\mathrm{ns}}$ \\
$\mathrm{Y} \times \mathrm{B}$ & 2 & $0.08^{\mathrm{ns}}$ & $0.08^{\mathrm{ns}}$ & $0.11^{\mathrm{ns}}$ & $0.05^{\mathrm{ns}}$ & $0.74^{\mathrm{ns}}$ & $0.08^{\mathrm{ns}}$ & $0.11^{\mathrm{ns}}$ \\
$\mathrm{V} \times \mathrm{C}$ & 3 & $1.44^{* *}$ & $1.44^{* *}$ & $9.11^{* *}$ & $11.22^{* *}$ & $3.18^{* *}$ & $1.44^{* *}$ & $9.11^{* *}$ \\
$\mathrm{~V} \times \mathrm{B}$ & 2 & $0.61^{*}$ & $0.61^{*}$ & $4.69^{* *}$ & $8.62^{* *}$ & $2.75^{* *}$ & $0.61^{*}$ & $4.69^{* *}$ \\
$\mathrm{C} \times \mathrm{B}$ & 6 & $0.28^{\mathrm{ns}}$ & $0.28^{\mathrm{ns}}$ & $1.83^{* *}$ & $1.70^{*}$ & $1.37^{*}$ & $0.28^{\mathrm{ns}}$ & $1.83^{* *}$ \\
$\mathrm{Y} \times \mathrm{V} \times \mathrm{C}$ & 3 & $0.02^{\mathrm{ns}}$ & $0.02^{\mathrm{ns}}$ & $0.53^{\mathrm{ns}}$ & $0.63^{\mathrm{ns}}$ & $2.07^{* *}$ & $0.02^{\mathrm{ns}}$ & $0.53^{\mathrm{ns}}$ \\
$\mathrm{Y} \times \mathrm{V} \times \mathrm{B}$ & 2 & $0.05^{\mathrm{ns}}$ & $0.05^{\mathrm{ns}}$ & $0.57^{\mathrm{ns}}$ & $0.36^{\mathrm{ns}}$ & $0.94^{\mathrm{ns}}$ & $0.05^{\mathrm{ns}}$ & $0.57^{\mathrm{ns}}$ \\
$\mathrm{Y} \times \mathrm{C} \times \mathrm{B}$ & 6 & $0.05^{\mathrm{ns}}$ & $0.05^{\mathrm{ns}}$ & $0.13^{\mathrm{ns}}$ & $0.24^{\mathrm{ns}}$ & $0.97^{*}$ & $0.05^{\mathrm{ns}}$ & $0.13^{\mathrm{ns}}$ \\
$\mathrm{V} \times \mathrm{C} \times \mathrm{B}$ & 6 & $0.71^{* *}$ & $0.71^{* *}$ & $1.97^{* *}$ & $2.89^{* *}$ & $4.00^{* *}$ & $0.71^{* *}$ & $1.97^{* *}$ \\
$\mathrm{Y} \times \mathrm{V} \times \mathrm{C} \times \mathrm{B}$ & 6 & $0.18^{\mathrm{ns}}$ & $0.18^{\mathrm{ns}}$ & $0.45^{\mathrm{ns}}$ & $0.72^{\mathrm{ns}}$ & $3.16^{* *}$ & $0.18^{\mathrm{ns}}$ & $0.45^{\mathrm{ns}}$ \\
$\mathrm{Error}$ & 92 & 0.20 & 0.20 & 0.44 & 0.59 & 0.46 & 0.20 & 0.44 \\
\hline $\mathrm{C} . \mathrm{V} .(\%)$ & - & 0.56 & 2.25 & 0.93 & 8.84 & 1.04 & 0.56 & 0.93 \\
\hline
\end{tabular}

ns, * and **: non significant and significant in $5 \%$ and $1 \%$ probability level, respectively

Table 5. Combined analysis of variance (ANOVA) of chlorophyll content and nitrogen, phosphorous and potassium content of grain in rice cultivar under chemical fertilizer and bio-fertilizer

\begin{tabular}{|c|c|c|c|c|c|c|c|c|c|}
\hline S.O.V. & DF & $\begin{array}{c}\text { Grain } \\
\text { length } \\
\text { before } \\
\text { cooking }\end{array}$ & $\begin{array}{l}\text { Grain } \\
\text { width } \\
\text { before } \\
\text { baking }\end{array}$ & $\begin{array}{c}\text { Grain } \\
\text { length } \\
\text { after } \\
\text { baking }\end{array}$ & $\begin{array}{c}\text { Grain } \\
\text { elongation }\end{array}$ & $\begin{array}{l}\text { Aroma } \\
\text { and } \\
\text { taste }\end{array}$ & $\begin{array}{c}\text { Gelatinization } \\
\text { temperature }\end{array}$ & $\begin{array}{c}\text { Gel } \\
\text { consistency }\end{array}$ & $\begin{array}{c}\text { Amylose } \\
\text { content }\end{array}$ \\
\hline Year $(Y)$ & 1 & $0.002^{\text {ns }}$ & $0.0001^{\mathrm{ns}}$ & $0.014^{\mathrm{ns}}$ & $0.006^{\mathrm{ns}}$ & $0.01^{\mathrm{ns}}$ & $0.02^{\mathrm{ns}}$ & $126.79^{* *}$ & $0.92^{*}$ \\
\hline Y (R) & 4 & 0.31 & 0.010 & 0.26 & 0.019 & 0.15 & 0.07 & 35.25 & 0.27 \\
\hline Variety (V) & 1 & $0.11^{\mathrm{ns}}$ & $0.04^{*}$ & $0.73^{*}$ & $0.27^{\mathrm{ns}}$ & $0.004^{\mathrm{ns}}$ & $0.37^{* *}$ & $1235.41^{* * *}$ & $1.22^{* *}$ \\
\hline Chemical F. (C) & 3 & $0.23^{*}$ & $0.02^{\mathrm{ns}}$ & $4.03^{* *}$ & $3.14^{* *}$ & $0.34^{* *}$ & $0.05^{\mathrm{ns}}$ & $1699.58^{* * *}$ & $8.76^{* *}$ \\
\hline Bio-fertilizer (B) & 2 & $0.15^{*}$ & $0.01^{\mathrm{ns}}$ & $0.17^{\mathrm{ns}}$ & $0.16^{\mathrm{ns}}$ & $0.13^{\mathrm{ns}}$ & $0.06^{*}$ & $188.84^{* *}$ & $4.61^{* *}$ \\
\hline $\mathrm{Y} \times \mathrm{V}$ & 1 & $0.02^{\mathrm{ns}}$ & $0.002^{\mathrm{ns}}$ & $0.001^{\mathrm{ns}}$ & $0.01^{\mathrm{ns}}$ & $0.07^{\mathrm{ns}}$ & $0.01^{\mathrm{ns}}$ & $98.70^{* * *}$ & $0.01^{\mathrm{ns}}$ \\
\hline $\mathrm{Y} \times \mathrm{C}$ & 3 & $0.004^{\mathrm{ns}}$ & $0.001^{\mathrm{ns}}$ & $0.003^{\mathrm{ns}}$ & $0.01^{\mathrm{ns}}$ & $0.01^{\mathrm{ns}}$ & $0.01^{\mathrm{ns}}$ & $179.85^{* *}$ & $0.19^{\mathrm{ns}}$ \\
\hline $\mathrm{Y} \times \mathrm{B}$ & 2 & $0.0004^{\mathrm{ns}}$ & $0.001^{\mathrm{ns}}$ & $0.001^{\mathrm{ns}}$ & $0.0002^{\mathrm{ns}}$ & $0.01^{\mathrm{ns}}$ & $0.01^{\mathrm{ns}}$ & $14.37^{\mathrm{ns}}$ & $0.08^{\mathrm{ns}}$ \\
\hline $\mathrm{V} \times \mathrm{C}$ & 3 & $0.12^{\mathrm{ns}}$ & $0.083^{* *}$ & $4.51^{* *}$ & $4.74^{* *}$ & $2.76^{* *}$ & $0.65^{* *}$ & $1097.41^{* * *}$ & $11.40^{* *}$ \\
\hline $\mathrm{V} \times \mathrm{B}$ & 2 & $0.16^{*}$ & $0.001^{\mathrm{ns}}$ & $2.76^{* *}$ & $1.60^{* *}$ & $1.28^{* *}$ & $0.10^{*}$ & $540.19^{* *}$ & $0.28^{\mathrm{ns}}$ \\
\hline $\mathrm{C} \times \mathrm{B}$ & 6 & $0.55^{* *}$ & $0.008^{\mathrm{ns}}$ & $1.53^{* *}$ & $1.43^{* *}$ & $0.46^{* *}$ & $0.04^{\mathrm{ns}}$ & $295.99^{* *}$ & $2.02^{* *}$ \\
\hline $\mathrm{Y} \times \mathrm{V} \times \mathrm{C}$ & 3 & $0.003^{\mathrm{ns}}$ & $0.0002^{\text {ns }}$ & $0.006^{\mathrm{ns}}$ & $0.002^{\mathrm{ns}}$ & $0.07^{\mathrm{ns}}$ & $0.11^{* *}$ & $123.72^{* * *}$ & $0.18^{\mathrm{ns}}$ \\
\hline $\mathrm{Y} \times \mathrm{V} \times \mathrm{B}$ & 2 & $0.003^{\mathrm{ns}}$ & $0.0002^{\text {ns }}$ & $0.007^{\mathrm{ns}}$ & $0.004^{\mathrm{ns}}$ & $0.02^{\mathrm{ns}}$ & $0.02^{\mathrm{ns}}$ & $33.12^{\mathrm{ns}}$ & $0.02^{\text {ns }}$ \\
\hline $\mathrm{Y} \times \mathrm{C} \times \mathrm{B}$ & 6 & $0.003^{\mathrm{ns}}$ & $0.0004^{\mathrm{ns}}$ & $0.004^{\mathrm{ns}}$ & $0.01^{\mathrm{ns}}$ & $0.03^{\mathrm{ns}}$ & $0.01^{\mathrm{ns}}$ & $39.05^{*}$ & $0.03^{\mathrm{ns}}$ \\
\hline $\mathrm{V} \times \mathrm{C} \times \mathrm{B}$ & 6 & $0.19^{* *}$ & $0.022^{\mathrm{ns}}$ & $2.23^{* *}$ & $1.81^{* *}$ & $0.97^{* *}$ & $0.20^{* *}$ & $848.88^{* * *}$ & $2.10^{* *}$ \\
\hline $\mathrm{Y} \times \mathrm{V} \times \mathrm{C} \times \mathrm{B}$ & 6 & $0.004^{\mathrm{ns}}$ & $0.0004^{\mathrm{ns}}$ & $0.001^{\mathrm{ns}}$ & $0.01^{\mathrm{ns}}$ & $0.11^{\mathrm{ns}}$ & $0.01^{\mathrm{ns}}$ & $77.12^{* *}$ & $0.05^{\mathrm{ns}}$ \\
\hline Error & 92 & 0.06 & 0.01 & 0.17 & 0.18 & 0.08 & 0.03 & 14.14 & 0.16 \\
\hline C.V. $(\%)$ & - & 3.34 & 5.83 & 3.70 & 11.50 & 16.50 & 4.77 & 4.46 & 2.13 \\
\hline
\end{tabular}

$\mathrm{ns}, *$ and $* *$ : non significant and significant in $5 \%$ and $1 \%$ probability level, respectively

Triple mean comparison by slice interaction showed that the least brown rice for $c v$. Tarom Hashemi was achieved with application of $75 \%$ recommended potassium. However, brown rice was highest at another level of mineral and biological fertilizer (Table 6). In $c v$. Tarom Mahalli, most brown rice was measured with $75 \%$ recommended phosphorous usage and 10 ton manure consumption and with $75 \%$ recommended potassium and 8 ton vermi-compost usage. The least brown rice for $c v$. 
Tarom Mahalli was observed at all mineral and biological fertilizer levels. The least hard husk for $c v$. Tarom Hashemi was observed with $100 \%$ NPK recommended and 10 ton manure consumption and with $75 \%$ recommended phosphorous. Moreover, minimum hard husk was observed at $75 \%$ recommended potassium and 8 ton vermicompost per hectare. The softest husk for $c v$. Tarom Hashemi was obtained with $100 \%$ mineral fertilizer and 10 ton manure per hectare. Softest husk for $c v$. Tarom Mahalli was obtained with $100 \%$ recommended NPK and 10 ton manure per hectare. The least soft husk for $c v$. Tarom Hashemi was obtained with $75 \%$ recommended nitrogen rate. For $c v$. Tarom Mahalli, the least soft husk was observed with $75 \%$ recommended PK rates along with 8 ton vermi-compost consumption and control treatment (Table 6).

Table 6. Mean comparison of cultivar, and chemical and biological fertilizers on the number of total, filled and blank spikelet and some technological parameters of rice by slice interaction

\begin{tabular}{|c|c|c|c|c|c|c|c|c|c|c|}
\hline Interaction & $\begin{array}{c}\text { Number of } \\
\text { spikelet per } \\
\text { panicle }\end{array}$ & $\begin{array}{c}\text { Number of } \\
\text { filled } \\
\text { spikelet per } \\
\text { panicle }\end{array}$ & \begin{tabular}{|c}
$\begin{array}{c}\text { Number of } \\
\text { blank } \\
\text { spikelet per } \\
\text { panicle }\end{array}$ \\
\end{tabular} & $\begin{array}{c}\text { Brown } \\
\text { rice }(\%)\end{array}$ & $\begin{array}{l}\text { Hard } \\
\text { husk } \\
(\%)\end{array}$ & $\begin{array}{l}\text { White } \\
\text { rice } \\
(\%)\end{array}$ & $\begin{array}{l}\text { Soft } \\
\text { husk } \\
(\%)\end{array}$ & $\begin{array}{c}\text { Health } \\
\text { grain } \\
(\%)\end{array}$ & $\begin{array}{l}\text { Broken } \\
\text { grain } \\
(\%)\end{array}$ & $\begin{array}{c}\text { Milling } \\
\text { efficiency } \\
(\%)\end{array}$ \\
\hline $\mathrm{V}_{1} \mathrm{C}_{1} \mathrm{~B}_{1}$ & $113.63 \mathrm{a}$ & $105.63 \mathrm{a}$ & $8.00 \mathrm{bc}$ & 80.80 a & $19.20 \mathrm{~b}$ & $70.53 \mathrm{~b}$ & $10.27 \mathrm{a}$ & $63.89 \mathrm{~b}$ & $6.63 \mathrm{~b}$ & $70.53 \mathrm{c}$ \\
\hline $\mathrm{V}_{1} \mathrm{C}_{1} \mathrm{~B}_{2}$ & $112.16 \mathrm{a}$ & $104.57 \mathrm{a}$ & $7.60 \mathrm{c}$ & $80.63 \mathrm{a}$ & $19.37 \mathrm{ab}$ & $70.85 \mathrm{~b}$ & $9.78 \mathrm{ab}$ & $64.33 \mathrm{ab}$ & $6.53 \mathrm{~b}$ & $70.85 \mathrm{c}$ \\
\hline $\mathrm{V}_{1} \mathrm{C}_{1} \mathrm{~B}_{3}$ & $100.00 \mathrm{ab}$ & $89.95 \mathrm{bc}$ & $10.05 \mathrm{ab}$ & 80.59 a & $19.41 \mathrm{ab}$ & $71.48 \mathrm{ab}$ & $9.11 \mathrm{ab}$ & $65.31 \mathrm{a}$ & $6.17 \mathrm{bc}$ & $71.48 \mathrm{abc}$ \\
\hline $\mathrm{V}_{1} \mathrm{C}_{2} \mathrm{~B}_{1}$ & $101.80 \mathrm{ab}$ & $93.05 \mathrm{~b}$ & $8.75 \mathrm{~b}$ & $80.67 \mathrm{a}$ & $19.33 \mathrm{ab}$ & $71.72 \mathrm{ab}$ & $8.95 \mathrm{~b}$ & $64.66 \mathrm{ab}$ & $7.06 \mathrm{ab}$ & $71.72 \mathrm{abc}$ \\
\hline $\mathrm{V}_{1} \mathrm{C}_{2} \mathrm{~B}_{2}$ & $100.77 \mathrm{ab}$ & $91.70 \mathrm{bc}$ & $9.07 \mathrm{~b}$ & $80.16 a b$ & $19.84 \mathrm{a}$ & $73.38 \mathrm{a}$ & $6.79 \mathrm{c}$ & $65.91 \mathrm{a}$ & $7.47 \mathrm{a}$ & $73.38 \mathrm{a}$ \\
\hline $\mathrm{V}_{1} \mathrm{C}_{2} \mathrm{~B}_{3}$ & $94.72 \mathrm{~b}$ & $82.93 \mathrm{c}$ & $11.78 \mathrm{a}$ & $80.13 a b$ & $19.88 \mathrm{a}$ & $72.05 \mathrm{a}$ & $8.07 \mathrm{~b}$ & $65.47 \mathrm{a}$ & $6.59 \mathrm{~b}$ & $72.05 \mathrm{ab}$ \\
\hline $\mathrm{V}_{1} \mathrm{C}_{3} \mathrm{~B}_{1}$ & $99.54 \mathrm{ab}$ & $89.75 \mathrm{bc}$ & $9.79 \mathrm{~b}$ & $80.34 \mathrm{a}$ & $19.66 \mathrm{a}$ & $71.53 \mathrm{ab}$ & $8.82 \mathrm{~b}$ & $65.37 \mathrm{a}$ & $6.16 \mathrm{~b}$ & $71.53 \mathrm{abc}$ \\
\hline $\mathrm{V}_{1} \mathrm{C}_{3} \mathrm{~B}_{2}$ & $108.08 \mathrm{a}$ & $98.67 \mathrm{ab}$ & $9.41 \mathrm{~b}$ & 80.46 a & $19.54 \mathrm{a}$ & $72.32 \mathrm{a}$ & $8.13 \mathrm{bc}$ & $65.11 \mathrm{a}$ & $7.22 \mathrm{a}$ & $72.32 \mathrm{ab}$ \\
\hline $\mathrm{V}_{1} \mathrm{C}_{3} \mathrm{~B}_{3}$ & & $86.98 \mathrm{bc}$ & $10.63 \mathrm{ab}$ & $80.81 \mathrm{a}$ & $19.19 \mathrm{~b}$ & $72.23 \mathrm{a}$ & $8.58 \mathrm{~b}$ & $65.84 \mathrm{a}$ & $6.38 \mathrm{~b}$ & \\
\hline $\mathrm{V}_{1} \mathrm{C}_{4} \mathrm{~B}_{1}$ & $101.47 \mathrm{ab}$ & $92.47 \mathrm{~b}$ & $9.00 \mathrm{~b}$ & $80.50 \mathrm{a}$ & $19.50 \mathrm{a}$ & $71.07 \mathrm{ab}$ & $9.43 \mathrm{ab}$ & $65.01 \mathrm{a}$ & $6.06 \mathrm{bc}$ & $71.07 \mathrm{abc}$ \\
\hline $\mathrm{V}_{1} \mathrm{C}_{4} \mathrm{~B}_{2}$ & $104.10 \mathrm{ab}$ & & & & $20.18 \mathrm{a}$ & $71.25 \mathrm{ab}$ & $8.57 \mathrm{~b}$ & & & $71.25 \mathrm{abc}$ \\
\hline $\mathrm{V}_{1} \mathrm{C}_{4} \mathrm{~B}_{3}$ & 97.94 b & $86.90 \mathrm{bc}$ & $11.04 \mathrm{a}$ & $79.66 \mathrm{~b}$ & $20.35 \mathrm{a}$ & $71.79 \mathrm{ab}$ & $7.87 \mathrm{bc}$ & $65.58 \mathrm{a}$ & $6.21 \mathrm{bc}$ & $71.79 \mathrm{abc}$ \\
\hline $\mathrm{V}_{2} \mathrm{C}_{1} \mathrm{~B}_{1}$ & $106.78 \mathrm{a}$ & $99.58 \mathrm{a}$ & $7.19 \mathrm{~b}$ & $79.44 \mathrm{~b}$ & $20.56 \mathrm{a}$ & \begin{tabular}{|l|}
$71.41 \mathrm{a}$ \\
\end{tabular} & $8.03 \mathrm{bc}$ & $65.12 \mathrm{a}$ & $6.29 \mathrm{~b}$ & $71.41 \mathrm{a}$ \\
\hline $\mathrm{V}_{2} \mathrm{C}_{1} \mathrm{~B}_{2}$ & $107.38 \mathrm{a}$ & 99.99 a & $7.39 \mathrm{~b}$ & $79.46 \mathrm{~b}$ & $20.54 \mathrm{a}$ & $70.64 \mathrm{ab}$ & $8.82 \mathrm{~b}$ & $64.87 \mathrm{ab}$ & $5.77 \mathrm{bc}$ & $70.64 \mathrm{~b}$ \\
\hline $\mathrm{V}_{2} \mathrm{C}_{1} \mathrm{~B}_{3}$ & $102.75 \mathrm{ab}$ & $91.25 \mathrm{ab}$ & $11.50 \mathrm{a}$ & $79.72 \mathrm{ab}$ & $20.28 \mathrm{ab}$ & $71.01 \mathrm{a}$ & $8.71 \mathrm{~b}$ & $63.09 \mathrm{~b}$ & $7.93 \mathrm{a}$ & $71.01 \mathrm{~b}$ \\
\hline $\mathrm{V}_{2} \mathrm{C}_{2} \mathrm{~B}_{1}$ & $104.25 \mathrm{a}$ & $94.35 \mathrm{a}$ & $9.91 \mathrm{ab}$ & $79.73 \mathrm{ab}$ & $20.27 \mathrm{ab}$ & $71.50 \mathrm{a}$ & $8.23 \mathrm{~b}$ & $64.49 \mathrm{ab}$ & $7.01 \mathrm{ab}$ & $71.50 \mathrm{a}$ \\
\hline $\mathrm{V}_{2} \mathrm{C}_{2} \mathrm{~B}_{2}$ & $103.86 \mathrm{a}$ & $95.57 \mathrm{a}$ & $8.30 \mathrm{ab}$ & $79.95 \mathrm{ab}$ & $20.05 \mathrm{ab}$ & $69.74 \mathrm{~b}$ & $10.21 \mathrm{a}$ & $63.77 \mathrm{~b}$ & $5.98 \mathrm{bc}$ & $69.74 \mathrm{~b}$ \\
\hline $\mathrm{V}_{2} \mathrm{C}_{2} \mathrm{~B}_{3}$ & & $82.35 \mathrm{~b}$ & & $79.47 \mathrm{~b}$ & $20.53 \mathrm{a}$ & $69.65 \mathrm{~b}$ & $9.82 \mathrm{ab}$ & $63.54 \mathrm{~b}$ & $6.12 \mathrm{~b}$ & $69.65 \mathrm{~b}$ \\
\hline $\mathrm{V}_{2} \mathrm{C}_{3} \mathrm{~B}_{1}$ & $96.46 \mathrm{ab}$ & $87.50 \mathrm{ab}$ & $8.96 \mathrm{ab}$ & $80.00 \mathrm{a}$ & $20.00 \mathrm{ab}$ & $71.21 \mathrm{a}$ & $8.79 \mathrm{~b}$ & $64.63 \mathrm{ab}$ & $6.58 \mathrm{~b}$ & $71.21 \mathrm{a}$ \\
\hline $\mathrm{V}_{2} \mathrm{C}_{3} \mathrm{~B}_{2}$ & $99.24 \mathrm{ab}$ & $87.96 \mathrm{ab}$ & $11.28 \mathrm{a}$ & $79.56 \mathrm{~b}$ & $20.45 \mathrm{a}$ & $71.70 \mathrm{a}$ & $7.86 \mathrm{c}$ & $65.40 \mathrm{a}$ & $6.30 \mathrm{~b}$ & $71.70 \mathrm{a}$ \\
\hline $\mathrm{V}_{2} \mathrm{C}_{3} \mathrm{~B}_{3}$ & $97.38 \mathrm{ab}$ & $86.43 \mathrm{ab}$ & $10.95 \mathrm{a}$ & $79.72 \mathrm{ab}$ & $20.29 \mathrm{ab}$ & $71.56 \mathrm{a}$ & $8.16 \mathrm{~b}$ & $65.82 \mathrm{a}$ & $5.74 \mathrm{c}$ & $71.56 \mathrm{a}$ \\
\hline $\mathrm{V}_{2} \mathrm{C}_{4} \mathrm{~B}_{1}$ & $107.08 \mathrm{a}$ & $97.40 \mathrm{a}$ & $9.68 \mathrm{ab}$ & $79.48 \mathrm{~b}$ & $20.52 \mathrm{a}$ & $71.04 \mathrm{ab}$ & $8.45 \mathrm{~b}$ & $64.16 \mathrm{ab}$ & $6.87 \mathrm{~b}$ & $71.04 \mathrm{~b}$ \\
\hline $\mathrm{V}_{2} \mathrm{C}_{4} \mathrm{~B}_{2}$ & $107.31 \mathrm{a}$ & $96.51 \mathrm{a}$ & $10.81 \mathrm{a}$ & $80.21 \mathrm{a}$ & $19.79 \mathrm{~b}$ & $71.33 \mathrm{a}$ & $8.88 \mathrm{~b}$ & $64.59 \mathrm{ab}$ & $6.74 \mathrm{~b}$ & $71.33 \mathrm{ab}$ \\
\hline $\mathrm{V}_{2} \mathrm{C}_{4} \mathrm{~B}_{3}$ & $92.73 \mathrm{~b}$ & $82.06 \mathrm{~b}$ & $10.66 \mathrm{a}$ & $79.77 \mathrm{ab}$ & $20.23 \mathrm{ab}$ & $71.79 \mathrm{a}$ & $7.98 \mathrm{c}$ & $65.41 \mathrm{a}$ & $6.38 \mathrm{~b}$ & $71.79 \mathrm{a}$ \\
\hline
\end{tabular}

Values within a column followed by same letter are not significantly different at $\mathrm{LSD}(\mathrm{P} \leq 0.05)$

$\mathrm{V}_{1}$ and $\mathrm{V}_{2}$ : Tarom Hashemi and Tarom Mahalli cultivars, respectively

$\mathrm{C}_{1}, \mathrm{C}_{2}, \mathrm{C}_{3}$, and $\mathrm{C}_{4}: 100 \%$ recommended NPK, $75 \%$ recommended nitrogen with $100 \% \mathrm{PK}, 75 \%$ recommended phosphorous with $100 \%$ NK, and $75 \%$ recommended potassium with $100 \%$ NP, respectively

$\mathrm{B}_{1}, \mathrm{~B}_{2}$, and $\mathrm{B}_{3}: 0$ and 8 ton vermi-compost per hectare and 10 ton of rotted manure per hectare, respectively

According to these findings, the lowest white rice for $c v$. Tarom Hashemi was obtained with $100 \%$ recommended NPK along with 10 ton manure and 8 ton vermi-compost consumption per hectare. For $c v$. Tarom Mahalli, the least white rice was obtained with $75 \%$ recommended nitrogen and 8 ton vermi-compost consumption and control treatment. However, whitest rice was obtained for both cultivars at all other levels of mineral 
fertilizer and bio-fertilizer (Table 6). Moreover, for $c v$. Tarom Hashemi the lowest health grain was obtained with $100 \%$ recommended NPK and 10 ton manure consumption per hectare. Furthermore, for $c v$. Tarom Mahalli the least healthy grain was produced at $100 \%$ recommended NPK applied along with bio-fertilizer control treatment and $75 \%$ recommended nitrogen and vermi-compost consumption and control treatment. However, higher rates of health grain were observed at other levels of mineral fertilizer and biofertilizer for both cultivars (Table 6). Least broken grain for $c v$. Tarom Hashemi was observed at $75 \%$ potassium along with vermi-compost consumption. Moreover, lowest broken grain was obtained with $75 \%$ phosphorous applied along with bio-fertilizer control level. Maximum broken grain for $c v$. Tarom Hashemi cultivar was observed at $75 \%$ recommended nitrogen and phosphorous applied with vermi-compost consumption. Furthermore, for $c v$. Tarom Mahalli maximum broken grain was obtained with manure consumption along with $75 \%$ recommended phosphorous (Table 6). In terms of milling efficiency, for $c v$. Tarom Hashemi maximum efficiency was obtained with $75 \%$ recommended nitrogen and vermi-compost consumption. For $c v$. Tarom Mahalli, maximum milling efficiency was achieved at different levels of mineral and biological fertilizers. The least milling efficiency for $c v$. Tarom Hashemi cultivar was produced with recommended $100 \%$ NPK along with cow manure and vermi-compost consumption. For $c v$. Tarom Mahalli, least conversion efficiency was observed for $100 \%$ recommended NPK and $75 \%$ recommended nitrogen along with vermi-compost consumption and control treatment (Table 6).

Based on the finding of mean comparison of cultivar, mineral fertilizer, and biofertilizer by slice interaction, least grain length before baking for $c v$. Tarom Hashemi cultivar was observed with $100 \%$ recommended NPK applied with vermi-compost consumption and control treatment. The lowest grain length before baking for $c v$. Tarom Mahalli was obtained with $75 \%$ recommended potassium usage and manure consumption. The highest grain length before baking for both cultivars was measured in other levels of mineral fertilizer and bio-fertilizer (Table 6). Moreover, maximum grain length after baking for $c v$. Tarom Hashemi was measured with $75 \%$ recommended nitrogen and bio-fertilizer control level along with 75\% recommended phosphorous usage and manure consumption at $12.48 \mathrm{~mm}$. Minimum grain length after baking for $c v$. Tarom Hashemi was calculated AT other levels of mineral and biological fertilizer levels. Furthermore, the least grain length after baking for $c v$. Tarom Mahalli was observed with $100 \%$ recommended NPK along with manure and vermi-compost consumption. Maximum grain length after baking for $c v$. Tarom Mahalli was observed at other levels of mineral and biological fertilizers (Table 7).

In terms of grain elongation, for $c v$. Tarom Hashemi cultivar maximum grain elongation was measured with $75 \%$ recommended nitrogen in bio-fertilizer control level along with $75 \%$ recommended phosphorous and mineral consumption at $4.92 \mathrm{~mm}$. The lowest grain elongation for $c v$. Tarom Hashemi was obtained with $75 \%$ recommended phosphorous and bio-fertilizer control treatment. For $c v$. Tarom Mahalli cultivar, least grain elongation was measured with $100 \%$ recommended NPK along with manure consumption. Maximum grain elongation for $c v$. Tarom Mahalli was obtained with $75 \%$ recommended nitrogen and vermi-compost consumption, $75 \%$ recommended phosphorous at all three levels of bio-fertilizer, and 75 recommended potassium along with manure and vermi-compost consumption at 4.52 and 4.27 (Table 7). Moreover, maximum aroma for $c v$. Tarom Hasemi was obtained with $75 \%$ of nitrogen recommended and control treatment along with $75 \%$ recommended phosphorous and 
vermi-compost consumption. Maximum aroma for $c v$. Tarom Mahalli was observed with $75 \%$ recommended potassium in bio-fertilizer control level (Table 7).

Table 7. Mean comparison of cultivar, and chemical and biological fertilizers on the number of total, filled and blank spikelet and some technological parameters of rice by slice interaction

\begin{tabular}{|c|c|c|c|c|c|c|c|}
\hline Interaction & $\begin{array}{l}\text { Grain length } \\
\text { before baking }\end{array}$ & $\begin{array}{l}\text { Grain length } \\
\text { after baking }\end{array}$ & $\begin{array}{c}\text { Grain } \\
\text { elongation }\end{array}$ & $\begin{array}{c}\text { Aroma and } \\
\text { taste }\end{array}$ & $\begin{array}{c}\text { Gelatinization } \\
\text { temperature }\end{array}$ & $\begin{array}{c}\text { Gel } \\
\text { consistency }\end{array}$ & $\begin{array}{l}\text { Amylose } \\
\text { content }\end{array}$ \\
\hline $\mathrm{V}_{1} \mathrm{C}_{1} \mathrm{~B}_{1}$ & $7.59 \mathrm{a}$ & $11.40 \mathrm{ab}$ & $3.81 \mathrm{ab}$ & $1.96 \mathrm{ab}$ & $3.56 \mathrm{a}$ & $95.78 \mathrm{a}$ & $17.72 \mathrm{bc}$ \\
\hline $\mathrm{V}_{1} \mathrm{C}_{1} \mathrm{~B}_{2}$ & $7.11 \mathrm{~b}$ & $10.77 \mathrm{~b}$ & $3.67 \mathrm{ab}$ & $1.91 \mathrm{ab}$ & $3.63 \mathrm{a}$ & $77.33 \mathrm{c}$ & $18.19 \mathrm{~b}$ \\
\hline $\mathrm{V}_{1} \mathrm{C}_{1} \mathrm{~B}_{3}$ & $7.13 \mathrm{~b}$ & $10.86 \mathrm{~b}$ & $3.73 \mathrm{ab}$ & $1.67 \mathrm{ab}$ & $3.35 \mathrm{a}$ & $76.99 \mathrm{c}$ & $17.44 \mathrm{c}$ \\
\hline $\mathrm{V}_{1} \mathrm{C}_{2} \mathrm{~B}_{1}$ & $7.43 \mathrm{a}$ & $11.25 \mathrm{ab}$ & $3.82 \mathrm{ab}$ & $1.90 \mathrm{ab}$ & $3.27 \mathrm{ab}$ & 95.09 a & $19.28 \mathrm{a}$ \\
\hline $\mathrm{V}_{1} \mathrm{C}_{2} \mathrm{~B}_{2}$ & $7.26 \mathrm{a}$ & $10.86 \mathrm{~b}$ & $3.59 \mathrm{ab}$ & $1.24 \mathrm{~b}$ & $3.27 \mathrm{ab}$ & $84.58 \mathrm{~b}$ & $17.92 \mathrm{bc}$ \\
\hline $\mathrm{V}_{1} \mathrm{C}_{2} \mathrm{~B}_{3}$ & $7.36 \mathrm{a}$ & $12.11 \mathrm{a}$ & $4.75 \mathrm{a}$ & $2.26 \mathrm{a}$ & $3.29 \mathrm{ab}$ & $93.05 \mathrm{ab}$ & $19.48 \mathrm{a}$ \\
\hline $\mathrm{V}_{1} \mathrm{C}_{3} \mathrm{~B}_{1}$ & $7.56 \mathrm{a}$ & $12.48 \mathrm{a}$ & $4.92 \mathrm{a}$ & $1.98 \mathrm{ab}$ & $3.39 \mathrm{ab}$ & $86.43 \mathrm{~b}$ & $18.93 \mathrm{ab}$ \\
\hline $\mathrm{V}_{1} \mathrm{C}_{3} \mathrm{~B}_{2}$ & $6.96 \mathrm{ab}$ & $10.75 \mathrm{~b}$ & $3.79 \mathrm{ab}$ & $2.36 \mathrm{a}$ & $3.26 \mathrm{ab}$ & $93.10 \mathrm{ab}$ & $17.93 \mathrm{bc}$ \\
\hline $\mathrm{V}_{1} \mathrm{C}_{3} \mathrm{~B}_{3}$ & $7.29 \mathrm{a}$ & $10.50 \mathrm{~b}$ & $3.21 \mathrm{~b}$ & $1.62 \mathrm{ab}$ & $3.07 \mathrm{~b}$ & $87.32 \mathrm{~b}$ & $18.48 \mathrm{ab}$ \\
\hline $\mathrm{V}_{1} \mathrm{C}_{4} \mathrm{~B}_{1}$ & $6.96 \mathrm{ab}$ & $9.90 \mathrm{~b}$ & $2.94 \mathrm{ab}$ & $1.67 \mathrm{ab}$ & $3.26 \mathrm{ab}$ & $87.79 \mathrm{~b}$ & $19.06 \mathrm{ab}$ \\
\hline $\mathrm{V}_{1} \mathrm{C}_{4} \mathrm{~B}_{2}$ & $7.46 \mathrm{a}$ & $10.33 \mathrm{~b}$ & $2.88 \mathrm{ab}$ & $1.13 \mathrm{~b}$ & $3.37 \mathrm{ab}$ & $77.32 \mathrm{c}$ & $18.78 \mathrm{ab}$ \\
\hline $\mathrm{V}_{1} \mathrm{C}_{4} \mathrm{~B}_{3}$ & $7.71 \mathrm{a}$ & $10.74 \mathrm{~b}$ & $3.03 \mathrm{ab}$ & $1.16 \mathrm{~b}$ & $3.38 \mathrm{ab}$ & $92.73 \mathrm{ab}$ & $18.54 \mathrm{ab}$ \\
\hline $\mathrm{V}_{2} \mathrm{C}_{1} \mathrm{~B}_{1}$ & $7.36 \mathrm{a}$ & $10.17 \mathrm{~b}$ & $2.80 \mathrm{~b}$ & $1.09 \mathrm{~b}$ & $3.17 \mathrm{~b}$ & $50.38 \mathrm{e}$ & $19.95 \mathrm{a}$ \\
\hline $\mathrm{V}_{2} \mathrm{C}_{1} \mathrm{~B}_{2}$ & $7.08 \mathrm{~b}$ & $10.43 \mathrm{~b}$ & $3.36 \mathrm{ab}$ & $1.60 \mathrm{ab}$ & $3.21 \mathrm{~b}$ & $69.60 \mathrm{~cd}$ & $18.93 \mathrm{~b}$ \\
\hline $\mathrm{V}_{2} \mathrm{C}_{1} \mathrm{~B}_{3}$ & $7.20 \mathrm{ab}$ & $10.72 \mathrm{ab}$ & $3.52 \mathrm{ab}$ & $1.68 \mathrm{ab}$ & $3.32 \mathrm{ab}$ & $88.46 \mathrm{~b}$ & $19.04 \mathrm{ab}$ \\
\hline $\mathrm{V}_{2} \mathrm{C}_{2} \mathrm{~B}_{1}$ & $7.46 \mathrm{a}$ & $10.73 \mathrm{ab}$ & $3.27 \mathrm{ab}$ & $1.16 \mathrm{~b}$ & $3.67 \mathrm{a}$ & $87.79 \mathrm{~b}$ & $19.69 \mathrm{a}$ \\
\hline $\mathrm{V}_{2} \mathrm{C}_{2} \mathrm{~B}_{2}$ & $7.50 \mathrm{a}$ & $11.58 \mathrm{a}$ & $4.08 \mathrm{a}$ & $1.93 \mathrm{ab}$ & $3.54 \mathrm{ab}$ & $89.57 \mathrm{~b}$ & $19.32 \mathrm{ab}$ \\
\hline $\mathrm{V}_{2} \mathrm{C}_{2} \mathrm{~B}_{3}$ & $7.63 \mathrm{a}$ & $11.18 \mathrm{ab}$ & $3.55 \mathrm{ab}$ & $1.61 \mathrm{ab}$ & $3.63 \mathrm{a}$ & $94.38 \mathrm{a}$ & $19.82 \mathrm{a}$ \\
\hline $\mathrm{V}_{2} \mathrm{C}_{3} \mathrm{~B}_{1}$ & $7.38 \mathrm{a}$ & $11.49 \mathrm{a}$ & $4.11 \mathrm{a}$ & $1.87 \mathrm{ab}$ & $3.37 \mathrm{ab}$ & $81.32 \mathrm{bc}$ & $17.20 \mathrm{c}$ \\
\hline $\mathrm{V}_{2} \mathrm{C}_{3} \mathrm{~B}_{2}$ & $7.57 \mathrm{a}$ & $11.79 \mathrm{a}$ & $4.22 \mathrm{a}$ & $1.68 \mathrm{ab}$ & $3.47 \mathrm{ab}$ & $74.77 \mathrm{bcd}$ & $17.17 \mathrm{c}$ \\
\hline $\mathrm{V}_{2} \mathrm{C}_{3} \mathrm{~B}_{3}$ & $7.25 \mathrm{ab}$ & $11.49 \mathrm{a}$ & $4.24 \mathrm{a}$ & $1.72 \mathrm{ab}$ & $3.56 \mathrm{ab}$ & $62.23 \mathrm{~d}$ & $18.78 \mathrm{~b}$ \\
\hline $\mathrm{V}_{2} \mathrm{C}_{4} \mathrm{~B}_{1}$ & $7.13 \mathrm{~b}$ & $11.64 \mathrm{a}$ & $4.52 \mathrm{a}$ & $1.86 \mathrm{ab}$ & $3.78 \mathrm{a}$ & $91.26 \mathrm{ab}$ & $18.81 \mathrm{~b}$ \\
\hline $\mathrm{V}_{2} \mathrm{C}_{4} \mathrm{~B}_{2}$ & $7.35 \mathrm{a}$ & $11.61 \mathrm{a}$ & $4.27 \mathrm{a}$ & $1.93 \mathrm{ab}$ & $3.31 \mathrm{ab}$ & $92.32 \mathrm{ab}$ & $17.57 \mathrm{c}$ \\
\hline $\mathrm{V}_{2} \mathrm{C}_{4} \mathrm{~B}_{3}$ & $7.58 \mathrm{a}$ & $10.83 \mathrm{ab}$ & $3.25 \mathrm{ab}$ & $2.61 \mathrm{a}$ & $3.28 \mathrm{ab}$ & $95.13 \mathrm{a}$ & $17.70 \mathrm{c}$ \\
\hline
\end{tabular}

Values within a column followed by same letter are not significantly different at LSD $(\mathrm{P} \leq 0.05)$

$\mathrm{V}_{1}$ and $\mathrm{V}_{2}$ : Tarom Hashemi and Tarom Mahalli cultivars, respectively

$\mathrm{C}_{1}, \mathrm{C}_{2}, \mathrm{C}_{3}$, and $\mathrm{C}_{4}: 100 \%$ recommended NPK, $75 \%$ recommended nitrogen with $100 \% \mathrm{PK}, 75 \%$ recommended phosphorous with $100 \% \mathrm{NK}$, and $75 \%$ recommended potassium with $100 \% \mathrm{NP}$, respectively

$\mathrm{B}_{1}, \mathrm{~B}_{2}$, and $\mathrm{B}_{3}$ : 0 and 8 ton vermi-compost per hectare and 10 ton of rotted manure per hectare, respectively

According to these findings, maximum gelatinization temperature for $c v$. Tarom Hshemi cultivar was measured at $100 \%$ recommended NPK applied at all three levels of bio-fertilizer. The least gelatinization temperature was calculated at $75 \%$ recommended phosphorous in bio-fertilizer control treatment. Highest gelatinization temperature for $c v$. Tarom Mahalli was obtained with $75 \%$ recommended nitrogen in manure consumption and control treatment along with $75 \%$ recommended potassium and manure consumption at 3.78. Minimum gelatinization temperature for $c v$. Tarom Mahalli was observed with $100 \%$ recommended NPK along with manure and vermicompost consumption at 3.17 and 3.21 (Table 7). In terms of gel consistency for $c v$. Tarom Hashemi cultivar, the highest amount was observed with 100 of NPK and $75 \%$ recommended nitrogen and manure consumption at 95.78 and $95.09 \mathrm{~mm}$. Lowest gel 
consistency for $c v$. Tarom Hshemi was observed with $75 \%$ recommended nitrogen with vermi-compost usage at bio-fertilizer control level along with $75 \%$ recommended potassium and vermi-compost consumption at $77.32 \mathrm{~mm}$. Moreover, the most gel consistency for $c v$. Tarom Mahalli cultivar was calculated with $75 \%$ recommended nitrogen in bio-fertilizer control treatment and 75\% recommended potassium in biofertilizer control level at $95.13 \mathrm{~mm}$. Least gel consistency for $c v$. Tarom Mahalli cultivar was measured at $100 \%$ recommended NPK and manure consumption. In terms of amylose content in $c v$. Tarom Hashemi cultivar the maximum rates was obtained with $75 \%$ recommended nitrogen with manure consumption and control treatment. Least Amylose content was observed with $100 \%$ recommended NPK applied in biofertilizer control treatment. For $c v$. Tarom Mahalli, maximum amylose content was produced with 100\% recommended NPK and manure consumption, and $75 \%$ recommended nitrogen along with manure usage and control treatment. Least amylose content was obtained with $75 \%$ recommended phosphorous with manure and vermicompost consumption and $75 \%$ recommended potassium with vermi-compost usage and control treatment at 17.57 and $17.70 \%$ (Table 7). In fact, coherence in the shape and paddy yield is the first factor in quality corroboration. Farmers consider these factors for the cultivation of new cultivars.

In general, biological fertilizers should be used instead of mineral fertilizers. Despite improving performance, chemical fertilizers lead to increased salinity and soil and water contamination. They also reduce the stability of the agricultural systems due to the use of chemical chemicals. If the use of organic fertilizer for more years which caused improves soil structure, prevents water and soil pollution, and leads to increased system stability because they increase soil organic matter. The only problem with the use of organic fertilizers is the low rate of decomposition of these materials: however, by using effective microorganisms and increasing the population of microbial degrading soil this too can be solved. In addition, researches shows that the use of manure instead of chemical fertilizer saves up to $25 \%$ in current costs, which adds to the sustainability of the cultivation system (Khaliq et al., 2006). According to the results of application of nitrogen fertilizer on rice growth parameters, the number of panicles per square meter, the total number of spikelet per panicle, and the number of blank spikelet per panicle increased, but the number of filled spikelet per panicle decreased (Jafari et al., 2013a, b). This is important because of the effects of the sink-source in the yield components (Jafari et al., 2013 b). Moreover, in another study on the rice plant, it was found that plant height, panicle length, and third inter-node length increased by increasing the nitrogen fertilizer. Moreover, the fourth inter-node length increased with increase in nitrogen: this also caused more lodging (Dastan et al., 2012).

Chemical fertilizers significant effect world food production and are one of the most important components in today's agriculture. Predictions show that more than $50 \%$ of agricultural production relies on the use of chemical fertilizers (Fixon and West, 2002). Conventional agricultural practices in today's world do not have an acceptable success rate of resource management, and rely excessively on artificial inputs and the use of ancillary energy inputs such as fertilizers and chemical pesticides to create unsustainable agro-ecosystems (Roberts, 2008). Biological fertilizers, including cow manure fertilizers, can increase soil water retention capacity, reduce tensions, and stress (Macilwain, 2004), increase soil microbial diversity (Oehl et al., 2004), improve soil physical structure (Pulleman et al., 2003), and prevent soil erosion (Pinamonti, 1998). This increases supply of part of the nutrition needed for the plant (Turgut et al., 2005), 
improves plant growth and yield (Kramer et al., 2002), and increases the quality of the product (Gilesm, 2004). Also, the development of soil fertility and soil quality, especially in the case of systems with low inputs, requires organic inputs to the soil (Palm et al., 2001). Moreover, Siavoshi et al. (2014) reported that biological fertilizer compared to chemical fertilizer in rice production improved qualitative traits and increases protein yield and paddy yield.

Field trials of fertilization and cultivar on the grain yield of rice (Oryza sativa L.) with two fertilizer levels and 18 modern varieties carried by Li et al. (2016) showed that variation among cultivars had a considerable impact on rice grain yield. Close correlations were observed between grain yield and effective panicles and dry matter production. Harvest index the ratio of grain weight to total plant weight, is an important trait associated with dramatic increases in crop yields. Most progress in improving HI occurred following the introduction of semi-dwarf traits into rice in the 1960s (Sinclair, 1998). However, the scope for continued HI increase in modern rice cultivars was limited by the need for maintaining sufficient leaf area and stem biomass for interception of solar radiation, physical support and storage of assimilate and $\mathrm{N}$ used in grain filling (Cassman, 1999; Cassman et al., 1998). Yang et al. (2006) demonstrated that post-anthesis dry matter production of high-yield rice differs between different yield categories. Experimental results in China on the effects of manure and chemical fertilizers on barley showed that the use of manure had similar (or even greater) effect on chemical fertilizers. Grain appearance is important for determining the quality of rice and its marketfriendliness. Grain length before cooking is one of the qualitative factors of the rice grain. Rice grains increase in size after cooking: if an increase in length takes place without an increase in thickness is considered a positive factor for cultivars. Rice-cooking traits are mainly determined by the properties of starch, which comprises up to $90 \%$ of the endosperm of white rice and consists of two amylose and amylopectin components. High amylose rice is completely separated after drying and is hardened after cooling. Most rice with low amylose content has a spikelet with a minimum amount of blank grain and a number of broken grains (Uphoff, 2011). Measurement of the gel consistency complements the amylose test, which indicates the amount of rice cooked gel movement. Gel consistency also shows the exact hardness and adhesion of cooked rice. The determination of endosperm starch gelatinization temperature is an important test for determining the quality of baking in rice. The temperature of the gelatinized starch is an important quality factor that is related to the cooking time and the nature of cooked rice: in fact, higher the gelatinization temperature, the higher the ranking of rice in terms of qualitative categorization. Studying the effects of genotype and environment on bread making quality in wheat, Dencic et al. (2011) showed that both cultivar and cultivar by environment interaction had significant effect on all quality traits. In addition, the variances of quality traits associated with the genetic factors of cultivars were generally larger than those of cultivars by environmental interaction effects.

\section{Conclusion}

According to the finding highest paddy yield occurs with cultivar and bio-fertilizer interaction involving vermi-compost and manure usage for both cultivars. Moreover, the highest paddy yield produced with $100 \%$ recommended NPK and vermi-compost consumption. Based on the results, the highest amount of gel consistency for $c v$. Tarom Hashemi cultivar was observed with 100 of NPK and $75 \%$ of recommended nitrogen 
and manure consumption. The maximum amylose content for $c v$. Tarom Mahalli was produced with $100 \%$ NPK recommended and manure consumption. Therefore, it can be concluded that by the integrated consumption of mineral and biological fertilizers, farmers can significantly reduce impacts of chemical fertilizers on the environment, and improve the qualitative and the quantitative parameters of rice cultivars with biological fertilizers. Hence, in the future researchers should be investigating the multiple consumptions of bio-fertilizer and chemical fertilizer for achieving sustainable agriculture.

\section{REFERENCES}

[1] Cassman, K. G. (1999): Ecological intensification of cereal production systems: yield potential, soil quality and precision agriculture. - Proceedings of the National Academy of Sciences 96: 5952-5959.

[2] Cassman, K. G., Peng, S., Olk, D. D., Ladha, J. K., Reicharolt, W., Dobermann, A., Singh, U. (1998): Opportunities for increased nitrogen use efficiency from improved resource management in irrigated rice systems. - Field Crops Research 56: 37-39.

[3] Dastan, S., Siavoshi, M., Zakavi, D., Ghanbari Malidarreh, A., Yadi, R., Ghorbannia, E., Nasiri, A. (2012): Application of nitrogen and silicon rates on morphological and chemical lodging related characteristics in rice (Oryza sativa) North of Iran. - Journal of Agriculture Science 4(6): 12-18.

[4] Dencic, S., Mladenov, N., Kobiljski, B. (2011): Effects of genotype and environment on bread making quality in wheat. - International Journal of Plant Production 5(1): 71-81.

[5] Deshpande, H., Devasenapathy, P. (2010): Effect of different organic sources of nutrients and green manure on growth and yield parameters of rice (Oryza sativa L.) grown under lowland condition. - Crop Research 41(1-3): 1-5.

[6] Elekhtyar, N. M., Metwally, T. F., Nour El-Din, M. (2016): Evaluation of bio-NPK and compost tea on seedling vigor and yield of rice. - Proceedings of First International Conference of Applied Microbiology. Egypt, March 1-3, 2016.

[7] FAO (2016): Faostat-Trade/Crops and Livestock Products. http://faostat3.fao.org/browse/T/TP/E [15 April 2016].

[8] Fixon, P. E. West, F. B. (2002): Nitrogen fertilizers: meeting contemporary challenges. AMBIO 31: 169-176.

[9] Ghosh, A., Sharma, A. R. (1999): Effect of combined use of organic manure and nitrogen fertilizer on the performance of rice under flood-prone lowland condition. - J. Agric. Sci. Cambridge 132: 461-465.

[10] Gilesm, J. (2004): Is organic food better for us? - Nat. (Lond.) 428: 796-797.

[11] Jafari, H., Dastan, S., Moshtaghian, M. R., Mohammadi, B., Valaei, L. (2013a): Effects of weeds control and nitrogen application on weeds and rice characteristics in Iranian paddy field. - Electronic Journal of Biology 9(4): 77-83.

[12] Jafari, H., Dastan, S., Nasiri, A. R., Valaei, L., Jafaripour, R. (2013b): Nitrogen and silicon application facts on rice growth parameters. - Electronic Journal of Biology 9(4): $72-76$.

[13] Jogloy et al. (2006): Kaentawan (Helianthus tuberosus L.): a new energy crop. - Khon Kaen Agricult. J. 34(2): 92-103.

[14] Khaliq, A., Abbasi, M. K., Hussain, T. (2006): Effect of integrated use of organic and inorganic nutrient sources with effective microorganisms (EM) on seed cotton yield in Pakistan. - Bio-resource Technology 97: 967-972.

[15] Kramer, A. W., Doane, T. A., Horwath, W. R., Van Kessel, C. (2002): Combining fertilizer and organic inputs to synchronize $\mathrm{N}$ supply in alternative cropping system in California. - Agriculture Ecosystem and Environmental 91: 233-243. 
[16] Li, X. K., Hill, P. W., Li, Y. C., Wang, Y., Ren, T., Cong, R. H., Lu, J. W. (2016): Physiological processes associated with high yield traits in modern rice varieties. International Journal of Plant Production 10(2): 221-231.

[17] Macilwain, C. (2004): Is organic farming better for the environment? - Nature 428: 797798.

[18] Metwally, T. F. (2015): Impact of organic materials combined with mineral nitrogen on rice growth, yield, grain quality and soil organic matter. - Int. J. Chem. Tech. Research 8(4): $1533-1542$.

[19] Ministry of Jihad-e-Agriculture of Iran. (2016): Annual Agricultural Statics. www.maj.ir.

[20] Oehl, F., Sieverding, E., Mäder, P., Duboi, D., Ineichen, K., Boller, T. and Wiemken, A. (2004): Impact of long-term conventional and organic farming on the diversity of arbuscular mycorrhizal fungi. - Oecologia 138: 574-583.

[21] Palm, A. C., Gachengo, C. N., Delve, R. J., Cadisch, G. and Giller, K. E. (2001): Organic inputs for soil fertility management in tropical agro-ecosystems: application of an organic resource database. - Agricultural Ecosystem Environment 8: 27-42.

[22] Pazouki, T. M., Ajam Noroui, H., Ghanbari Malidareh, A., Dadashi, M. R., Dastan, S. (2017): Energy and $\mathrm{CO}_{2}$ emission assessment of wheat (Triticum aestivum L.) production scenarios in central areas of Mazandaran province, Iran. - Applied Ecology and Environmental Research 15(4): 143-161.

[23] Pinamonti, F. (1998): Compost mulch effects on soil fertility, nutritional status and performance of grapevine. - Nutrition Cycling Agro-ecosystem 51: 239-248.

[24] Pishgar-Komleh, S. H., Sedeedpari, P., Rafiee, S. (2011): Energy and economic analysis of rice production under different farm levels in Guilan province of Iran. - Energy 36: $5824-5831$.

[25] Pulleman, M. A., Jongmans, J., Bouma, J. (2003): Effects of organic versus conventional arable farming on soil structure and organic matter dynamics in a marine loam in the Netherlands. - Soil Use Manage 19: 157-165.

[26] Regmi, A. P., Ladha, J. K., Pathak, H., Pasuquin, E., Buenon, C., Dawe, D., Hobbs, P. R., Joshy, D., Maskey, S. L., Pandey, S. P. (2002): Yield and soil fertility trends in a 20-year rice-rice-wheat experiment in Nepal. - Soil Sci. Soc. Am. Journal 66: 857-867.

[27] Rekhi, R. S., Singh, R., Goel, R. K., Singh, J. (2004): Crop yield, disease incidence, and insect pest attack in relation to $\mathrm{N}$ dynamics in rice. - IRRN 29: 65-67.

[28] Roberts, T. L. (2008): Improving nutrient use efficiency. - Turkish Journal of Agriculture 32: $177-182$.

[29] Roy, D. K., Singh, B. P. (2006): Effect of level and time of nitrogen application with and without vermicompost on yield, yield attributes and quality of malt barley (Hordeum vulgare). - Indian Journal of Agronomy 51: 40-42.

[30] Siavoshi, M., Dastan, S., Yadi, R. (2014): Relationship between organic fertilizers and some parameters of rice grain quality. - Advances in Environmental Biology 8(17): 521530.

[31] Sinclair, T. R. (1998): Historical changes in harvest index and crop nitrogen accumulation. - Crop Science 38: 638-643.

[32] Turgut, I., Bilgili, U., Duman, A., Acikgoz, E. (2005): Effect of green manure on the yield of sweet corn. - Agronomy Sustainable Development 25: 1-5.

[33] Uphoff, N. (2011): The system of rice intensification: an alternate civil society innovation. - Journal of Technology Assessment in Theory and Practice 20(2): 45-51.

[34] Xu, K., Lei, Y., Choi, J. K. (2008): Kiitricha minuta n. sp., a peculiar hypotrichous ciliate (Ciliophora Spirotrichea) from the Yellow Sea. - Journal of Eukaryot Microbiol 55: 201-206.

[35] Yang, J., Du, Y., Wu, C., Liu, L., Wang, Z., Zhu, Q. (2006): Growth and development characteristics of super-high-yielding mid-season Japonica rice. - Scientia Agricola 39: $1336-1345$. 